\title{
Understanding the effects of ion irradiation using nanoindentation techniques.
}

\author{
Christopher D Hardie ${ }^{a, b}$, Steve G Roberts ${ }^{b}$ and Andy J Bushby ${ }^{c}$ \\ ${ }^{a}$ EURATOM/CCFE Association, Culham Centre for Fusion Energy (CCFE) Abingdon, Oxfordshire OX14 \\ 3DB, UK \\ ${ }^{\mathrm{b}}$ Department of Materials, University of Oxford, Oxford, OX1 3PH, United Kingdom \\ 'School of Engineering and Materials Science, Queen Mary University of London, Mile End Road, \\ London, E1 4NS, United Kingdom
}

\section{ABSTRACT}

The effects of ion irradiation in materials for research are usually limited to a shallow surface layer of the order of one micrometre in depth. Determining the mechanical properties of such irradiated materials requires techniques with high spatial resolution. Nanoindentation is a relatively simple method for investigating these shallow layers with the advantage that statistically rich data sets for elastic and plastic property values can be generated. However, interpretation of the results requires and understanding of the material response, including the extent of the plastic zone with respect to the irradiated layer, pile-up or sink-in of material around the indentation site that affect the calculated contact area and hence derived mechanical property values. $\mathrm{An} \mathrm{Fe}^{+}$self-irradiated Fe12\%Cr alloy was investigated with three different indenter tip geometries, a Cube corner, Berkovich and 10 micron radius indenter. Sharp indenters provide sufficiently small plastic zones to be contained within the irradiated layer but pop-in events and pile-up need to be taken into account for correct interpretation of the mechanical properties of the irradiated material. 


\section{INTRODUCTION AND BACKGROUND}

Ion implantation can quickly produce high levels of displacement damage without inducing radioactivation of samples and is therefore a popular tool to investigate irradiation damage in solids. Several microstructural and chemical characterisation methods such as transmission electron microscopy (TEM), atom probe tomography (APT) and positron annihilation spectroscopy (PAS), require volumes of material which are inherently small relative to ion irradiated surface layers which are typically a few microns in thickness. Thus the limited irradiated volume produced by implantation presents little or no additional challenges when using these techniques. In contrast, there are few methods available for the characterisation of mechanical properties from such small volumes of material, and analyses regarding best practise and validity of using these techniques for irradiated materials are scarce. There have been several investigations which have used nanoindentation as a means to measure the effects of ion irradiation on mechanical properties [112]. The majority of investigations have been conducted using a Berkovich tip geometry and include various methods such as load-unload $[1,2]$ and continuous stiffness measurement (CSM) [8-11]. Within all investigations there is a lack of consistency or justification of indentation parameters used and various methods for data interpretation; this makes cross-comparison of such experiments difficult. Therefore there is a requirement to understand and optimise nanoindentation techniques specifically for the measurement of mechanical properties of shallow ion irradiated layers.

The work described in this paper investigates and compares several nanoindentation techniques available for the analysis of an $800 \mathrm{~nm}$ damage layer of ion-irradiated $\mathrm{Fe} 12 \% \mathrm{Cr}$ alloy implanted at the National Ion Beam Centre, Surrey UK. Data produced from a Continuous Stiffness Measurement (CSM) nanoindentation technique with cube corner and Berkovich pyramidal tips and a partialunload technique with a spherical tip are directly compared and critically evaluated. Micro- 
mechanical techniques offer an alternative method for testing ion irradiated layers; in comparison to nanoindentation, these methods are more intensive on resources, time and expertise and are not described here. Testing using several micro-cantilever techniques on the same material have also been conducted and are reported in Ref. [13] in this journal edition.

\section{Experimental Details}

\subsection{Material and Sample Preparation}

The material and irradiation details are identical to that reported in [10], where full details have been given. Briefly, an Fe12\%Cr alloy was manufactured by Cambridge Metals Crystals and Oxides (CMCO) with final cold rolling into sheet at a thickness of approximately $1 \mathrm{~mm}$. A sample was cut into a rectangle sample of approximately $4 \times 8 \mathrm{~mm}$, and then annealed within an evacuated sealed quartz silica tube at $830^{\circ} \mathrm{C}+/-10^{\circ} \mathrm{C}$ for 72 hours to develop a large equiaxed grain structure. A series of lapping stages using SiC abrasive papers from FEPA P120 to P4000 grades was used to produce a smooth surface with a thin layer of polishing damage. Finally a chemo-mechanical polish with a colloidal silica suspension $(0.05 \mu \mathrm{m})$ was used to provide a surface with minimal polishing damage and of a quality suitable for electron backscattered diffraction (EBSD). Grain diameters were determined by EBSD to be in the range of 20 to $450 \mu \mathrm{m}$, with a mean grain size of $189 \mu \mathrm{m}$.

\subsection{Ion Implantation}

The alloy was irradiated with $\mathrm{Fe}^{+}$ions at $320^{\circ} \mathrm{C}$ to an average dose of $6.18 \mathrm{dpa}(0-800 \mathrm{~nm}$ depth). Two implantation energies of $2 \mathrm{MeV}$ and subsequently $0.5 \mathrm{MeV}$ were used in an attempt to produce a uniform distribution of damage with depth. The ion beam and environmental conditions for the implantation are shown in table 1. Calculations of damage with depth into the sample surface are 
presented in figure 1, these were produced using the SRIM 2013 code [14] and a threshold displacement energy of 40eV for iron [15].

Table 1 - Ion beam conditions at the Surrey Ion Beam Centre (UK).

\begin{tabular}{|l|c|c|}
\hline Implantation Energy & $0.5 \mathrm{MeV}$ & $2 \mathrm{MeV}$ \\
\hline Implantation Dose (ions/cm ${ }^{2}$ ) & $1.5 \times 10^{15}$ & $3 \times 10^{15}$ \\
\hline Radiation Damage (avg.) & \multicolumn{2}{|c|}{$6.18 \mathrm{dpa}$} \\
\hline Radiation Damage (max.) & \multicolumn{2}{|c|}{$8.36 \mathrm{dpa}$} \\
\hline
\end{tabular}

Figure 2 shows a TEM cross-section of the radiation damage layer at the sample surface (as reported in ref. [10]). The micrograph includes the protective Pt layer deposited in the FIB before foil thinning, a region of dense dislocation loops forming the damage layer and the underlying un-irradiated substrate. It is evident that the visible damage extends to a depth no more than $600 \mathrm{~nm}$ at the sample surface which is slightly less than that predicted by the SRIM calculations. The sample clamp used during ion-irradiation produced a sample with areas both exposed to and shielded from the beam. The region of the sample exposed to the beam was clearly visible in secondary electron images after implantation as it was darker in contrast compared to the un-implanted region of the sample. This enabled the FIB milling of marker lines which were visible under an optical microscope, at the boundary between implanted and un-implanted regions. This enabled mechanical testing adjacent to the boundary of the implanted and un-implanted regions within same grain and provided the advantage of eliminating differences in observed mechanical properties due to any anisotropy associated with crystallographic orientation, variation in the quality of polishing and sample mounting conditions. 


\subsection{Nanoindentation Techniques}

For nanoindentation testing the alloy sample was mounted on an aluminium stub using a thin layer of Crystalbond $^{\circledR}$ resin. Three diamond indenter tip geometries were used for comparison of indentation response. These were a pyramidal cube corner tip with a centreline to face angle of $34.3^{\circ}$, a pyramidal Berkovich tip with centreline to face angle of $65.3^{\circ}$ and a spherical tip with a nominal radius of $10 \mu \mathrm{m}$. The geometry of pyramidal indenter tips are described by an area function, $A=f\left(h_{c}\right)$, and were calibrated by indentation of a reference fused silica sample with a known elastic modulus of 72GPa according to the methods described in ref. [16]. The spherical indenter was calibrated using the multiple reference material method [17].

Continuous Stiffness Method nanoindentation (CSM) [18] was carried out with the pyramidal tips using an MTS NANO Indenter XP fitted with the NANO CSM system (MTS NANO Oak Ridge Tennessee, USA). A small sinusoidal oscillation in the load signal measures stiffness dynamically during the indentation sequence and the corresponding displacement signal is monitored. Contact stiffness can be calculated by measuring the phase difference or the amplitude of the displacement signal, accounting for the response of the entire nanoindentation system by using a dynamic model (as given in ref. [16]).

The stiffness of the contact, $d P / d h$, has contributions from both the sample $(E)$ and the indenter tip $\left(E_{i}\right)$. This is described as the reduced modulus, $\left(E_{r}\right)$, which is calculated from the stiffness, $S$, of the initial part of the unloading curve and the area of contact between indentation tip and sample surface, $A$, by: 
$S=\frac{d P}{d h}=\frac{2}{\sqrt{\pi}} E_{r} \sqrt{A}$

where $P$ is the indentation load on the surface and $h$ is the indentation displacement into the surface. The sample modulus is therefore calculated by correcting for tip deformation by:

$\frac{1}{E_{r}}=\frac{\left(1-v^{2}\right)}{E}+\frac{\left(1-v_{i}^{2}\right)}{E_{i}}$

where the elastic modulus, $E_{i}$, and Poisson's ratio, $v_{i}$, for a diamond indenter are $1141 \mathrm{GPa}$ and 0.07 respectively [16].

The calculation of hardness also requires knowledge of the contact area and is defined as:

$H=\frac{P}{A}$

where contact area, $A$, is determined from the contact depth, $h_{c}$, and the calibrated area function of the indenter, $A=f\left(h_{c}\right)$.

Nanoindentation using the spherical indenter tip $(R=10 \mu \mathrm{m})$ was conducted using a UMIS 2000 instrument (CSIRO, Lindfield, NSW, Australia). This system directly measured the displacement of the indenter column and the deflection of the springs applying the load to the sample using two linear variable differential transducers (LVDT). A partial unloading technique was used, whereby the shape of the unloading curve was calculated using Hertzian contact mechanics for each load-partial unload data pair [19]. All indentations were conducted with 60 load-partial unload increments, with a partial unloading fraction of $75 \%$ of the total increment load $\left(P_{i}\right)$.

The depth of penetration, $h_{i}$, of a sphere of radius, $R$, into an elastic half space at a load, $P_{i}$, is defined as: 
$h_{i}=\left(\frac{9}{16}\right)^{\frac{1}{3}}\left(\frac{P_{i}}{E_{r}}\right)^{\frac{2}{3}}\left(\frac{1}{R}\right)^{\frac{1}{3}}$

$E_{r}$ is the reduced modulus and can be used to find the sample elastic modulus using the same equation as that for pyramidal tips. Similarly, hardness is calculated using the same equation as that for pyramidal tips, however the contact area is defined by the tip radius which was calibrated as a function of indentation depth using multiple references samples and according to the method described in ref. [17].

\subsection{Atomic Force Imaging}

In order to measure indentation sink-in or pile-up of material, surface topography maps of deformed regions surrounding indents were produced using atomic force microscopy (AFM) using a Park Autoprobe CP-II AFM with a multitask head. The microscope was fitted with a chip containing 3 beam-shaped cantilevers supplied by Mikromasch (NSC35/AIBS). The sharp tip at the end of each cantilever has a radius of curvature at the apex of typically $10 \mathrm{~nm}$. Proprietary software (Veeco, now Bruker) was used to control the microscope during imaging.

\subsection{Transmission Electron Microscopy (TEM)}

Transmission electron microscopy (TEM) specimen preparation was carried out using a lift-out technique using a Zeiss Auriga dual beam SEM-FIB; this method is described fully in ref. [20]. This technique enabled the production of cross-sectional specimens which included deformed material surrounding indents within both irradiated and un-irradiated regions of the sample. TEM imaging was conducted with a Phillips CM20 microscope with an operating voltage of 200kV. 


\section{Results and Discussion}

Important tip dependent parameters such as the size of the plastic zone, stress distribution and contact area must be considered for the interpretation of results. This involves a number of additional techniques for investigating material post indentation.

\subsection{Plastic Zone Size}

The volume of material which is deformed by elastic and plastic processes is an important parameter in the interpretation of indentation data and is particularly important for tests on thin films such as ion-implanted layers. There have been several investigations which model the distribution of stresses surrounding the indenter tip [21] and which examine plastic deformation directly [22-24]. The latter consists of several methods such as AFM for the observation of the plastic zone size laterally at the sample surface and/or FIB manufactured cross-sectional specimens for EBSD and TEM of deformation beneath the indenter tip. The development of a plastic zone surrounding a nano-scale indent is likely to be dependent on several factors such as the material, crystal orientation, tip geometry and indentation depth [22]. Therefore, the multiplication factors (ranging from 4 to 10) which relate indentation depth with the plastic zone size $[25,26]$ provide a somewhat simplified case and are of limited value for systems where the thickness of a layer/coating presents a significant limitation.

Cross-sectional TEM analysis of nanoindents produced with a range of depths and loads were conducted to determine the size and shape of the plastic zone surrounding the three different types of indenter tip. For all tips, indents were produced within the same grain to avoid differences in plasticity due to variations in crystallographic orientation. For each region, the indents of increasing depth were produced in a line so that a large foil containing cross-sections of all the indents could be manufactured in the FIB. Deformation below each pyramidal indent is produced by a flat surface of 
the tip on right and a tip edge on the left. Cross-sections of spherical indents were only produced in the irradiated material due to the large indentation pop-in events in the un-irradiated material. This indentation behaviour prevented plastic deformation occurring in the un-irradiated material at indentation depths less than $150 \mathrm{~nm}$, therefore a comparison with the irradiated material was not possible.

Typical cross-sectional TEM images of both irradiated and un-irradiated regions, showing the plastic zones surrounding indents produced by the cube corner, Berkovich and spherical tips, is presented in figure 3, figure 4 and figure 5 respectively.

At similar indentation depths the size of the plastic zone, observable by a network of dislocations, changes dramatically as a function of the tip depth-area ratio (the tip sharpness). For the pyramidal tips, the plastic zone size in irradiated material is suppressed in comparison to the un-irradiated material by the presence of the damaged layer. This effect is reduced at larger indentation depths where the plastic zone appears to penetrate through the damage layer into the underlying unirradiated material. In all cases it is evident that the deformation has developed into multiple regions, such that dislocations are formed into separate lobes where plastic strain has been accommodated by favourable slip systems. This evolving and irregular shape of deformation means that measurement of the plastic zone is difficult to define for such small indentations in a single crystal. Here we define it by a semi-circular area which includes all regions/lobes of dense dislocations in the TEM micrographs in figures 3-5. Figure 6 shows the evolution of plastic zone radius with indenter depth for all three tips in the irradiated and un-irradiated regions of the material. Again, data for spherical indentation in the un-irradiated material is missing due to large pop-in events and a fully elastic indentation in the depth range of interest. The increase in plastic zone size is close to linear 
for all three tips. The rate of increase is very similar for both pyramidal tips, yet the growth of plastic zone is far greater in for the spherical tip. The extent of the plastic zone is indicated in figure 6, which suggests that the plastic zone starts to extend beyond the irradiated layer at depths of $~ 50 \mathrm{~nm}$, $\sim 100 \mathrm{~nm}$ and $\sim 200 \mathrm{~nm}$ for the spherical, Berkovich and cube corner tips respectively. Despite this, the indentation response is dominated by the irradiated layer to larger indent depths, due to the large fraction of plastic zone remaining within the damage layer.

\subsection{Indentation Sink-in and Pile-up}

Before cross-sectioning, AFM scanning and SEM imaging of the indent impressions was conducted to investigate the evolution of sink-in or pile-up with indentation depth. Both sink-in and pile-up of material surrounding the indenter tip influence the actual contact area and can cause errors and/or artefacts in mechanical property measurements which are determined from the calculated contact area using the measured displacements and a calibrated tip area function.

The extent of sink-in or pile-up is closely related to the proximity of the plastic deformation in relation to the indenter tip. For indents in bulk materials, this behaviour is dependent on the ratio of elastic modulus to yield stress, $E / \sigma_{\text {yield }}$, and the work hardening coefficient, $n$, of the material (where $\sigma=K \epsilon^{n}$ ) $[27,28]$. In addition, pile-up/sink-in can be more prominent in indentation tests which include a thin coating on an underlying substrate [25]. Plastic deformation may take place in the substrate (away from the tip) for indentation in hard coatings on a soft substrate or adjacent to the tip for indentation in a soft coating on a hard substrate [29]. Thus, the substrate beneath thin damage layers produced by ion-implantation may influence the sink-in/pile-up behaviour during 
indentation. Differences in sink-in/pile-up properties between irradiated and un-irradiated materials may obscure the observation of irradiation-induced changes in mechanical properties.

The surface topography surrounding each indent was observed by AFM to investigate the evolution of pile-up with indentation depth. A typical 3D image of the surface topography surrounding a cube corner indent is given in figure $7 a$, which clearly shows the pile-up morphology corresponding to the three flat surfaces of the indenter tip. Line profiles were taken through the centre of each indent; a typical example of surface data from each line profile is shown in figure $7 b$. For pyramidal tip indentations, the position of the line profiles (as indicated on figure 7a) provided surface height data through an apex of the indentation (where two flat surfaces of the tip meet) and through the flat edge of the indentation with adjacent pile-up lobe. AFM data showing pile-up lobe sizes for indentation depths of $50-250 \mathrm{~nm}$ produced by both pyramidal tips are shown in figure 8 .

All indents produced by the pyramidal tips produced pile-up adjacent to the flat edges of the indenter tip and pile-up extended to greater heights in the irradiated material. Figure 9 shows line profiles taken through the deepest point in the residual impressions produced by spherical indentation in the irradiated material. In contrast to indentations produced by the pyramidal tips, sink-in was observed for indentations produced at low loads and pile-up only developed once the indent had reached a load of $50 \mathrm{mN}$.

For the spherical tip, the actual contact area was calculated by defining a new contact radius, $a$, identified by the point of inflection in the AFM profiles [30]. Measuring the contact area by the AFM line profiles for the pyramidal tips was attempted according to a method proposed by Kese et al. [31]. This approach assumed that the additional contact area was in the form of a semi-ellipse with a chord length equal to the flat edge of the indentation impression and a segment height equal to 
width measurements of the pile-up in contact with the tip. SEM images of the Berkovich and cube corner indents in the un-irradiated and irradiated material are shown in figure 10. These images show that the semi-ellipse assumption cannot be used for small, single crystal indents due to inhomogeneous plastic deformation and pile-up behaviour. The actual contact area accounting for the pile-up was evaluated by the direct measurement of area using SEM imaging as shown in figure 10, in a similar method to that proposed by Tsui et al. [29].

Figure 11 shows the ratio of actual contact area to the corner-to-corner measurement of contact area (no pile-up) for the pyramidal tips, and actual contact area to depth-sensing measurements for the spherical indents with increasing depth; this ratio represents sink-in for values $<1$ and pile-up for values $>1$. Data are shown for both irradiated and un-irradiated regions of the sample apart from the spherical indentations where indent impressions were limited to depths $>150 \mathrm{~nm}$ due to large pop-in events in the un-irradiated material.

The additional contact area due to pile-up $\left(A_{a c t u a l} / A_{c c}\right)$ surrounding the pyramidal tips was significant. For both pyramidal tips, $A_{a c t u a l} / A_{c c}$ in the un-irradiated material decreases as the indentation depth increases. For indentation depths from 50 to $250 \mathrm{~nm}, A_{\text {actual }} / A_{c c}$ produced by the cube corner and Berkovich tips range from $\sim 60$ to $20 \%$ and $\sim 30$ to $10 \%$. For the cube corner tip, $A_{\text {actual }} / A_{c c}$ in the irradiated material increases from $>30 \%$ to $>60 \%$ over the same depth range. Similarly, $A_{a c t u a l} / A_{c c}$ for the Berkovich tip increases with indentation depth to a depth between 50 and $200 \mathrm{~nm}$ and then decreases dramatically. For spherical indents, the material surrounding the indenter tip exhibited sink-in $\left(A_{a c t u a l} / A_{c c}<0\right)$, which increased with indentation depth.

The variation in contact area produced by pile-up/sink-in will cause an error of $E /\left(A_{\text {actual }} / A_{c c}\right)^{-1 / 2}$ in elastic modulus and $H / A_{a c t u a l} / A_{c c}$ in hardness. The next section will assess the effect of pile-up and sink-in in the mechanical property measurements produced using the depth sensing technique. 


\subsection{Indentation Data}

Figure 12 shows nanoindentation data produced by using the cube corner, Berkovich and spherical tips. For each tip arrays of indents were produced in the un-irradiated and irradiated regions of the same grain. The indentation data presented for the pyramidal tips is an average of $\sim 8$ indents and error bars plot the standard deviation. Due to the 'pop-in' nature of the spherical indents (discussed below), the data presented for the spherical tip is from a single indent. The total indentation depth $\left(h_{t}\right)$ was controlled to be $2000 \mathrm{~nm}$ for the pyramidal tips, which produced maximum loads $\left(P_{t}\right)$ of $25 \mathrm{mN}$ for cube corner indents and $138 \mathrm{mN}$ for Berkovich indents. Spherical indentation (using the UMIS 2000 instrument) was conducted under a load-control method with a maximum load $\left(P_{t}\right)$ of $35 \mathrm{mN}$; for indents which exhibited plastic deformation, this produced an indentation depth $\left(h_{t}\right)$ of approximately $400 \mathrm{~nm}$.

For all three tips, the indentation load at a given indentation depth was higher in the irradiated material; this translates to a higher hardness measurement, which tends towards that of the unirradiated material as the indentation depth increases. The spherical indentation in the un-irradiated material exhibited large elastic stresses and in the majority of cases the indentation response remained entirely elastic up to $35 \mathrm{mN}$ (shown on figure $12 \mathrm{~g}$ ); at this load the indentation depth was 150nm producing a mean pressure $\left(P_{m}\right)$ of up to $7.8 \mathrm{GPa}$ beneath the indenter tip.

For both pyramidal tips, the elastic modulus appears to be higher in the irradiated material; but as shown in figure 14 and discussed in a later section, this is entirely accounted for when using the data with the actual contact area which corrects the data for contributions from pile-up. The elastic modulus measured with the Berkovich tip decreases with indentation depth, which is likely to be 
caused by poor compliance in the sample mounting method (Crystalbond ${ }^{\circledR}$ ) which decreases the apparent contact stiffness at the high loads produced with the Berkovich geometry $(138 \mathrm{mN})$.

\subsubsection{Indentation Pop-in}

The elastic-plastic transition behaviour of indents produced by all three indenter tips can be observed in the load-depth data at low indentation depths in figure 13. In every case, the irradiated material exhibited a smooth transition from elastic to plastic deformation. In the un-irradiated material, the initiation of plastic deformation can be identified by a number of discrete jumps in the tip displacement known as pop-in events; these are produced by the nucleation of the first dislocations, relieving strain beneath the tip [32]. At very early indentation depths (before the first pop-in), all three tips are essentially spherical in shape due to blunting of the pyramidal tips and the load-depth data exhibit Hertzian contact behaviour (as plotted for the spherical tip in figure 12 and 13). The magnitude of the pop-ins are similar for the two pyramidal tips, however the smallest jump in tip displacement in the data produced by the spherical tip was significantly larger (from $58 \mathrm{~nm}$ to $151 \mathrm{~nm})$.

\subsubsection{Elastic Modulus}

As shown in figure 14 the apparent elastic modulus calculated by depth sensing data produced by both pyramidal tips is larger for the irradiated material. This is accounted for entirely when recalculating the same indent data with SEM measurements of the actual contact area (section 3.2). The additional area produced by pile-up $\left(A_{\text {actual }} / A_{c c}\right)$ increases the apparent elastic modulus by approximately 10 to $30 \%$. The elastic modulus measured with the spherical tip decreases with increasing load; this is entirely accounted for by the sink-in of material identified by area corrections from the AFM measurements discussed in section 3.2. Sink-in decreases the apparent elastic modulus by $\sim 30 \%$ at the highest load $(35 \mathrm{mN})$. Elastic modulus data from an indent which remained 
entirely elastic in the un-irradiated region are also plotted in figure 14. The elastic response of this indent is not affected by plastic sink-in and should therefore remain independent of depth. In contrast to the un-irradiated data which was in an elastic-plastic regime at a depth greater than $51 \mathrm{~nm}$, the measured elastic modulus remains close to the corrected value of $190 \mathrm{GPa}(+/-2.7 \mathrm{GPa})$. Data from fully elastic indents are subject to noise at indentation depths below 50nm and the total indentation depth is limited due to reaching the maximum load of $35 \mathrm{mN}$.

\subsubsection{Hardness}

Figure 15 shows the hardness measurements from the raw CSM data of a typical indent (for pyramidal tips), depth-sensing data taken at $P_{\max }$ for the indents with increasing depth and values corrected for the actual contact area accounting for pile-up/sink-in (section 3.2). In comparison to the corrected measurements, values for hardness using the depth sensing method were up to $41 \%$ higher for the cube corner tip, $84 \%$ higher for the Berkovich tip and $19 \%$ lower for the spherical tip. The corrected hardness values from 50 to $250 \mathrm{~nm}$ produced an irradiation hardening $(\Delta H)$ of $\sim 0.25 \mathrm{GPa}$ for cube corner data and $\sim 0.6 \mathrm{GPa}$ for Berkovich data. A value of irradiation hardening could not be directly calculated from the data produced by the spherical tip, due to the lack of plastic data until after pop-in at depths of approximately $151 \mathrm{~nm}$. The same indentation depth in the irradiated region corresponds to a plastic zone which has expanded well beyond the damage layer into the underlying un-irradiated material.

\subsection{Comparison of Nanoindentation Techniques}

A primary concern when using nanoindentation for mechanical property measurements of thin surface layers such as ion-implanted surfaces is the range of indentation depth which produce representative mechanical property data of the material within the layer. For the measurement of the $\sim 800 \mathrm{~nm}$ thick irradiated layer discussed here, the useful indentation depth range is limited due 
to high levels of noise at smaller depths and contribution from the underlying un-irradiated substrate at larger depths. Table 2 provides the corrected and un-corrected mechanical property data for the un-irradiated and irradiated material tested with all tips and both techniques used. The cross-sectional TEM analysis for all tips has enabled the measurement of plastic zone size with increasing indentation depth and has provided the information required for defining a depth range for all tip geometries.

Table 2 - Mechanical property data uncorrected and corrected for pile-up for all three indentation methods. Data averaged over a depth range of $100-250 \mathrm{~nm}$ for cube corner and $50-200 \mathrm{~nm}$ for Berkovich tips. Data taken at $\sim 5 \mathrm{mN}(52 \mathrm{~nm})$ for spherical indentation.

\begin{tabular}{|c|c|c|c|c|c|}
\hline \multicolumn{2}{|l|}{ HARDNESS } & \multicolumn{2}{|c|}{ Un-irradiated } & \multicolumn{2}{|c|}{ Irradiated } \\
\hline & & $\begin{array}{c}\text { Hardness } \\
\text { (GPa) }\end{array}$ & SD & $\begin{array}{c}\text { Hardness } \\
\text { (GPa) }\end{array}$ & SD \\
\hline \multirow{3}{*}{ Corrected } & Cube Corner (100-250nm) & 1.5 & 0.04 & 1.72 & 0.08 \\
\hline & Berkovich (50-200nm) & 1.31 & 0.15 & 1.9 & 0.15 \\
\hline & Spherical $(5 \mathrm{mN} / 52 \mathrm{~nm})$ & Fully Elastic & & 1.8 & \\
\hline \multirow{3}{*}{ Uncorrected } & Cube Corner (100-250nm) & 1.9 & 0.12 & 2.28 & 0.14 \\
\hline & Berkovich (50-200nm) & 1.88 & 0.11 & 3.03 & 0.08 \\
\hline & Spherical (5mN/52nm) & Fully Elastic & & 1.72 & \\
\hline
\end{tabular}

\begin{tabular}{|c|c|c|c|c|c|}
\hline \multicolumn{2}{|c|}{ ELASTIC MODULUS } & \multicolumn{2}{|c|}{ Un-irradiated } & \multicolumn{2}{|l|}{ Irradiated } \\
\hline & & $\begin{array}{c}\text { Elastic Modulus } \\
\text { (GPa) }\end{array}$ & SD & $\begin{array}{l}\text { Elastic Modulus } \\
\text { (GPa) }\end{array}$ & SD \\
\hline \multirow{3}{*}{ Corrected } & Cube Corner (100-250nm) & 152 & 11 & 157 & 6 \\
\hline & Berkovich (50-200nm) & 158 & 18 & 160 & 16 \\
\hline & Spherical (5mN/52nm) & & & 190 & - \\
\hline Uncorrected & Cube Corner (100-250nm) & 173 & 6 & 185 & 8 \\
\hline
\end{tabular}




\begin{tabular}{|l|l|c|c|c|c|}
\hline Berkovich (50-200nm) & 193 & 11 & 210 & 13 \\
\cline { 2 - 5 } & Spherical (5mN/52nm) & 192 & - & 209 & - \\
\hline
\end{tabular}

A significant limitation in the use of the spherical tip at smaller depths is set by the prominent pop-in events in the un-irradiated material, which prevent the measurement of plastic properties at depths less than at least $150 \mathrm{~nm}$.

As described in section 3.1 the expansion of the plastic zone is suppressed by the radiation damage in the irradiated layer. This correlates well with the observations of larger pile-up surrounding the pyramidal tips in section 3.2, where deformation is constrained closer to the tip compared to the unirradiated material (figure 8). As the indentation depth increases the plastic zone extends beyond the damage layer and the hardness tends towards that of the un-irradiated material (figure 12). A large fraction of the plastic zone beneath the Berkovich indentation extends beyond the damage layer at a depth of approximately $200 \mathrm{~nm}$. This is also indicated by the relative drop in the pile-up from 200 to $250 \mathrm{~nm}$ shown in figure 11 . The hardness measured in the irradiated material with the Berkovich tip tends towards the un-irradiated value at smaller depths compared to measurements for the cube corner tip. This may be caused by a combination of significant pile-up surrounding the cube corner with increasing depth and a smaller plastic zone in the irradiated material. Due to the large pop-in events, hardness data produced by the spherical tip are limited; however there is only a small difference in hardness between the un-irradiated and irradiated material (0.22GPa) evident after the pop-in event at an indentation depth of $150 \mathrm{~nm}$. As shown in figure 5, the plastic zone has extended well beyond the damage layer at spherical indentation depths greater than $\sim 100 \mathrm{~nm}$.

Pharr et al. [33] have shown that there are three potential sources of error in the measurement of mechanical properties using the CSM method at indentation depths $<100 \mathrm{~nm}$ : 
i. During CSM measurements, the NanoXP system records the mean load rather than the peak load. At low indentation depths, the oscillation in tip displacement can cause a relatively large oscillation in the load resulting in a lower mean load compared to the peak load.

ii. As described in section 2.3 the contact stiffness is calculated from the amplitude of the load and displacement signals $(\Delta P / \Delta h)$. This assumes that the unloading load-displacement curve is linear within the range of oscillation; however at low indentation depths the large oscillation in load may exhibit curvature in the unloading curve.

iii. At low indentation depths, the indenter tip and surface may not be in full contact throughout the entire displacement range of oscillation.

All three points above may result in a lower measured stiffness at low indentation depths. As shown in the raw data on figure 14, both pyramidal tips produced low elastic modulus measurements compared to standard Fe measurements, which increased with depth up to a consistent value at $\sim 50-100 \mathrm{~nm}$. This happens in both un-irradiated and irradiated material, thus cannot be caused by a reduction in stiffness associated with the pop-in events exhibited in the un-irradiated material.

The value of load over stiffness squared $\left(P / S^{2}\right)$ is a useful indentation parameter as it is independent of indentation depth and contact area, provided elastic modulus and hardness do not vary with indentation depth [18]. Therefore, the plot of $P / S^{2}$ versus depth can be used for the identification of potential sources of error in nanoindentation data. Figure 16 shows $P / S^{2}$ versus depth for indentation data produced in the un-irradiated region of the same grain in the $\mathrm{Fe} 12 \% \mathrm{Cr}$ sample. The spherical indentation data was not produced by a CSM method and the values for $P / S^{2}$, shown in figure 16 for comparison, are constant for the data available in the depth range of 151 to $400 \mathrm{~nm}$. The pyramidal tips exhibit higher values of $P / S^{2}$ at smaller indentation depths, which appear to stabilise at a depth of $\sim 150-200 \mathrm{~nm}$ for the cube corner tip and $\sim 50-75 \mathrm{~nm}$ for the 
Berkovich tip. At low depths, higher values of $P / S^{2}$ may be caused by a reduction in stiffness as a result of (ii) and (iii) described above. From equations 1 and $3, P_{t}=f(A)$ and $S=f\left(A^{\frac{1}{2}}\right)$, thus the resulting load oscillation as a fraction of total load $\left(P_{o s} / P_{t}\right)$ produced by the displacement oscillation $(h(\omega))$ of $2 \mathrm{~nm}$ decreases as indentation depth increases. Without knowledge of the exact tip shape for the first few tens of nanometres of indentation depth it is unclear how the effect of (ii) and (iii) would differ between the two pyramidal tips. As shown in figure 17, the ratio of load oscillation to total load $\left(P_{o s} / P_{t}\right)$ does not differ substantially between the two tips at low depths due to similar levels of blunting. For both tips, the value of $\left(P_{o s} / P_{t}\right)$ is greater than 1 for depths up to $30-40 \mathrm{~nm}$. It is likely that the tip is not in full contact at these indentation depths and may produce errors in the value of $P / S^{2}$ up to approximately 50nm.

The depth range where $P_{o S} / P_{t}>1$ cannot explain the decrease in the value of $P / S^{2}$ with depth produced by the cube corner tip. The non-linear behaviour may be caused by an indentation size effect (ISE) [34], which is likely to be more prominent in the data produced by the cube corner tip. The size of the plastic zone with indentation depth (figure 6) is smaller than that produced by the Berkovich and spherical tips and hardness data produced by the cube corner (figure 15) indicates that an ISE exists. For steel, values of hardness are commonly related to flow stress by diving by 3.03 [35], yet even with this reduction factor it is clear that all of the nanoindentation results reported here are affected by an ISE [34]. Because this affect is not well understood, the mechanical property data produced by nanoindentation is limited to relative comparison between irradiation conditions and materials, and provides little information on the macroscopic properties of the material. Despite identifying similar trends, the several methods which exist all have compromising characteristics which affect the absolute and relative values in the results. 


\section{Conclusion}

Nanoindentation offers a relatively simple means to produce consistent, statistically rich mechanical property data in shallow ion irradiated layers. In the present study nanoindentation has successfully measured the effect of ion-irradiation on the mechanical properties of an $\mathrm{Fe} 12 \% \mathrm{Cr}$ alloy, however the magnitude of irradiation-induced changes in mechanical properties is dependent on the indentation technique and tip geometry used.

Indentation with the cube corner tip provides the advantage of the smallest plastic zone for thin surface layers, however the difference in pile-up between the irradiated and un-irradiated material produces significant errors in the observed irradiation hardening if an area function, rather than direct contact area measurement is used. The Berkovich tip provides a good compromise between small pop-in events and exhibits moderate pile-up with a small plastic zone size. Thus, data produced by indentation with a Berkovich tip at depths $50-200 \mathrm{~nm}$ has been used here. Observable by an apparent increase in elastic modulus, pile-up also produces larger apparent irradiation hardening. This pile-up is caused by the restricted growth of the plastic zone in the damage layer; therefore indentation data which are uncorrected for pile-up are considered a measure of both irradiation hardening and irradiation-induced indentation pile-up.

Unfortunately, the prominent pop-in events produced by the spherical tip in the present investigation prevented a direct comparison between un-irradiated and irradiated material and may well present similar problems in other materials. Unlike the pyramidal tips, the geometry of the sphere results in the increase of strain from zero upwards analogous to conventional tensile testing. In cases where larger volumes of material are available, spherical indentation may offer the ability to provide mechanical property (stress-strain) data which provides more information regarding the deformation characteristics of the material and has greater engineering relevance. Moreover, indentation with multiple tip radii may provide an opportunity to measure the effect of test scale, 
which is inherent in the majority of small scale testing on irradiated materials and may therefore indicate mechanical properties at the macro-scale. 


\section{Acknowledgments}

The authors thank the Surrey lon Beam Centre, UK, for assistance with ion implantation. C.D. Hardie would also like to thank EPSRC and CCFE for funding in the form of an Industrial CASE studentship.

\section{References}

[1] P. Hosemann, C. Vieh, R. R. Greco, S. Kabra, J. A. Valdez, M. J. Cappiello, and S. A. Maloy. Nanoindentation on ion irradiated steels. J. Nucl. Mater. 389 (2009) 239-247.

[2] X. M. Liu, , M. Le Flem, J. L. Béchade, and I. Monnet. Nanoindentation investigation of heavy ion irradiated $\mathrm{Ti}_{3} \mathrm{Si}_{1} \mathrm{AlC}_{2}$. J. Nucl. Mater. 401 (2010) 149-153.

[3] C.D. Hardie, S.G. Roberts, A.J. Bushby, Mechanical Behavior of Ion-Irradiated Fe-Cr Alloys Investigated by Spherical Indentation, Proc MRS. 1424 (2012).

[4] A.J. Bushby, S.G. Roberts, C.D. Hardie, Nanoindentation investigation of ion-irradiated $\mathrm{Fe}-\mathrm{Cr}$ alloys using spherical indenters, J. Mat. Res. 27 (2012) 85-90.

[5] C. Heintze, C. Recknagel, F. Bergner, M. Hernández-Mayoral, and A. Kolitsch. Ion-irradiationinduced damage of steels characterized by means of nanoindentation. Nucl. Instrum. Meth. B 267, no. 8 (2009): 1505-1508.

[6] M. Ando, H. Tanigawa, S. Jitsukawa, T. Sawai, Y. Katoh, A. Kohyama, K. Nakamura, and H. Takeuchi. Evaluation of hardening behaviour of ion irradiated reduced activation ferritic/martensitic steels by an ultra-micro-indentation technique. J. Nucl. Mater. 307 (2002): 260-265.

[7] H. Ogiwara, A. Kohyama, H. Tanigawa, and H. Sakasegawa. Irradiation-induced hardening mechanism of ion irradiated JLF-1 to high fluences. Fusion Eng. Des. 81, no. 8 (2006): 1091-1097.

[8] R. Kasada, Y. Takayama, K. Yabuuchi, and A. Kimura. A new approach to evaluate irradiation hardening of ion-irradiated ferritic alloys by nano-indentation techniques. Fusion Eng. Des. 86, no. 9 (2011): 2658-2661.

[9] C. Shin, H. Jin, and M-W. Kim. Evaluation of the depth-dependent yield strength of a nanoindented ion-irradiated $\mathrm{Fe}-\mathrm{Cr}$ model alloy by using a finite element modeling. J. Nucl. Mater. 392, no. 3 (2009): 476-481.

[10] C.D. Hardie, S.G. Roberts, Nanoindentation of model Fe-Cr alloys with self-ion irradiation, J. Nucl. Mater. 433 (2013) 174-179.

[11] C.D. Hardie, C.A. Williams, S. Xu, S.G. Roberts, Effects of irradiation temperature and dose rate on the mechanical properties of self-ion implanted Fe and Fe-Cr alloys, J. Nucl. Mater. 439 (2013) $33-40$. 
[12] P. Hosemann, D. Kiener, Y. Wang, and S.A. Maloy. Issues to consider using nano indentation on shallow ion beam irradiated materials. J. Nucl. Mater. 425, no. 1 (2012): 136-139.

[13] D.E. Armstrong, C. Hardie, J. Gibson, Busby. Understanding the effects of ion irradiation using micro and nano-mechanical testing, 2014, in this special edition

[14] J.F. Ziegler, M.D. Ziegler, J.P. Biersack, SRIM - The stopping and range of ions in matter (2010), Nucl. Instrum. Methods Phys. Res. B. Beam Interact. Mater. Atoms. 268 (2010) 1818-1823.

[15] ASTM Standard ASTM E521 - 96(2009), Standard Practice for Neutron Radiation Damage Simulation by Charged-Particle Irradiation, ASTM International, West Conshohocken, PA, (2009).

[16] W.C. Oliver, G.M. Pharr, An improved technique for determining hardness and elastic modulus using load and displacement sensing indentation experiments., J Mater Res. 7 (1992) 1564-1583.

[17] T.T. Zhu, A.J. Bushby, D.J. Dunstan, Size effect in the initiation of plasticity for ceramics in nanoindentation, J. Mech. Phys. Solids. 56 (2008) 1170-1185.

[18] W.C. Oliver, G.M. Pharr, Measurement of hardness and elastic modulus by instrumented indentation: Advances in understanding and refinements to methodology, JMR. 19 (2004) 3-20.

[19] K.L. Johnson, Contact mechanics, Cambridge university press, 1987.

[20] R.M. Langford, C. Clinton, In situ lift-out using a FIB-SEM system, Micron. 35 (2004) 607-611.

[21] A. Bolshakov, W.C. Oliver, G.M. Pharr, Influences of stress on the measurement of mechanical properties using nanoindentation: Part II. Finite element simulations, J. Mater. Res. 11 (1996) 760768.

[22] C.L. Woodcock, D.F. Bahr, Plastic zone evolution around small scale indentations, Scr. Mater. 43 (2000) 783-788.

[23] N. Zaafarani, D. Raabe, R.N. Singh, F. Roters, S. Zaefferer, Three-dimensional investigation of the texture and microstructure below a nanoindent in a Cu single crystal using 3D EBSD and crystal plasticity finite element simulations, Acta Materialia. 54 (2006) 1863-1876.

[24] M. Rester, C. Motz, R. Pippan, The deformation-induced zone below large and shallow nanoindentations: A comparative study using EBSD and TEM, Phil Mag Lett. 88 (2008) 879-887.

[25] G.M. Pharr, W.C. Oliver, Measurement of thin film mechanical properties using nanoindentation, MRS Bulletin. 17 (1992) 28-33.

[26] H. Buckle, Use of the Hardness Test to Determine Other Material Properties, in: J.H. Westbrook, H. Conrad (Eds.), The Science of Hardeness Testing and Its Research Applications, American Society for Metals, Metals Park, Ohio. (1971) 453-494.

[27] A.C. Fischer-Cripps, Factors Affecting Nanoindentation Test Data, in: Nanoindentation, Springer Science, New York. (2011) 77-103. 
[28] K.W. McElhaney, J.J. Vlassak, W.D. Nix, Determination of indenter tip geometry and indentation contact area for depth sensing indentation experiments, J. Mater. Res. 13 (1997) 1300-1306.

[29] T.Y. Tsui, G.M. Pharr, Substrate effects on nanoindentation mechanical property measurement of soft films on hard substrates, J. Mater. Res. 14 (1998) 292-301.

[30] X. Hou, A. Bushby, N. Jennett, Direct measurement of surface shape for validation of indentation deformation and plasticity length-scale effects: a comparison of methods, Measurement Science and Technology. 21 (2010) 115105.

[31] K.O. Kese, Z.C. Li, B. Bergman, Method to account for true contact area in soda-lime glass during nanoindentation with the Berkovich tip, Materials Science and Engineering: A. 404 (2005) 1-8.

[32] D. Lorenz, A. Zeckzer, U. Hilpert, P. Grau, Pop-in effect as homogeneous nucleation of dislocations during nanoindentation, Phys. Rev. B. 67 (2003) 172101-1-172101-4.

[33] G.M. Pharr, J.H. Strader, W. Oliver, Critical issues in making small-depth mechanical property measurements by nanoindentation with continuous stiffness measurement, J. Mater. Res. 24 (2009) 653-666.

[34] W.D. Nix, H. Gao, Indentation size effects in crystalline materials: A law for strain gradient plasticity, J. Mech. Phys. Solids. 46 (1998) 411-425.

[35] J.T. Busby, M.C. Hash, G.S. Was, The relationship between hardness and yield stress in irradiated austenitic and ferritic steels, J. Nucl. Mater. 336 (2005) 267-278. 


\section{Tables and Figures}

Table 1 - Ion beam conditions at the Surrey Ion Beam Centre (UK).

\begin{tabular}{|l|c|c|}
\hline Implantation Energy & $0.5 \mathrm{MeV}$ & $2 \mathrm{MeV}$ \\
\hline Implantation Dose (ions/cm ${ }^{2}$ ) & $1.5 \times 10^{15}$ & $3 \times 10^{15}$ \\
\hline Radiation Damage (avg.) & \multicolumn{2}{|c|}{$6.18 \mathrm{dpa}$} \\
\hline Radiation Damage (max.) & \multicolumn{2}{|c|}{$8.36 \mathrm{dpa}$} \\
\hline
\end{tabular}


Table 2 - Mechanical property data uncorrected and corrected for pile-up for all three indentation methods. Data averaged over a depth range of $100-250 \mathrm{~nm}$ for cube corner and $50-200 \mathrm{~nm}$ for Berkovich tips. Data taken at $\sim 5 \mathrm{mN}(52 \mathrm{~nm})$ for spherical indentation.

\begin{tabular}{|c|c|c|c|c|c|}
\hline \multicolumn{2}{|l|}{ HARDNESS } & \multicolumn{2}{|c|}{ Un-irradiated } & \multicolumn{2}{|c|}{ Irradiated } \\
\hline & & $\begin{array}{c}\text { Hardness } \\
\text { (GPa) }\end{array}$ & SD & $\begin{array}{c}\text { Hardness } \\
\text { (GPa) }\end{array}$ & SD \\
\hline \multirow{3}{*}{ Corrected } & Cube Corner (100-250nm) & 1.5 & 0.04 & 1.72 & 0.08 \\
\hline & Berkovich (50-200nm) & 1.31 & 0.15 & 1.9 & 0.15 \\
\hline & Spherical $(5 \mathrm{mN} / 52 \mathrm{~nm})$ & Fully Elastic & & 1.8 & \\
\hline \multirow{3}{*}{ Uncorrected } & Cube Corner (100-250nm) & 1.9 & 0.12 & 2.28 & 0.14 \\
\hline & Berkovich (50-200nm) & 1.88 & 0.11 & 3.03 & 0.08 \\
\hline & Spherical (5mN/52nm) & Fully Elastic & & 1.72 & \\
\hline
\end{tabular}

\begin{tabular}{|c|c|c|c|c|c|}
\hline \multicolumn{2}{|c|}{ ELASTIC MODULUS } & \multicolumn{2}{|c|}{ Un-irradiated } & \multicolumn{2}{|l|}{ Irradiated } \\
\hline & & $\begin{array}{c}\text { Elastic Modulus } \\
\text { (GPa) }\end{array}$ & SD & $\begin{array}{l}\text { Elastic Modulus } \\
\text { (GPa) }\end{array}$ & SD \\
\hline \multirow{3}{*}{ Corrected } & Cube Corner (100-250nm) & 152 & 11 & 157 & 6 \\
\hline & Berkovich (50-200nm) & 158 & 18 & 160 & 16 \\
\hline & Spherical (5mN/52nm) & & & 190 & - \\
\hline \multirow{3}{*}{ Uncorrected } & Cube Corner (100-250nm) & 173 & 6 & 185 & 8 \\
\hline & Berkovich (50-200nm) & 193 & 11 & 210 & 13 \\
\hline & Spherical (5mN/52nm) & 192 & - & 209 & - \\
\hline
\end{tabular}




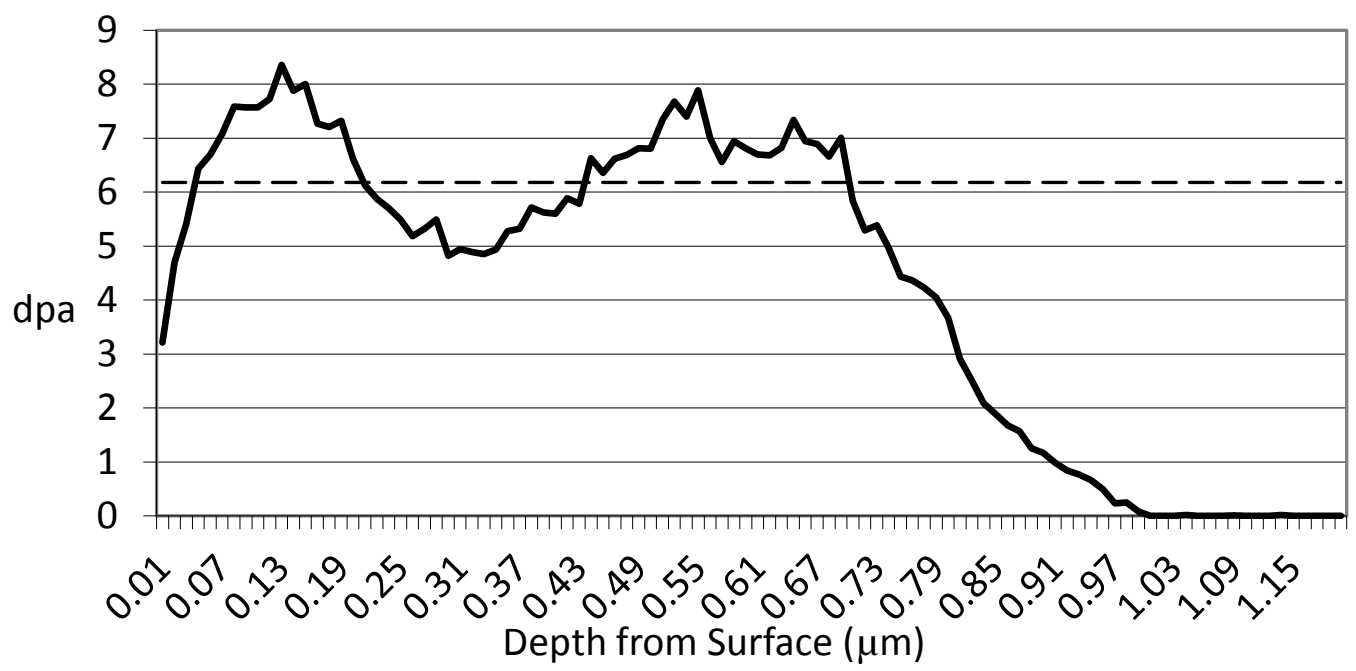

Figure 1 - Irradiation damage versus depth from the sample surface as calculated by SRIM with a displacement energy of $40 \mathrm{eV}$ for iron [15].

\section{$500 \mathrm{~nm}$}

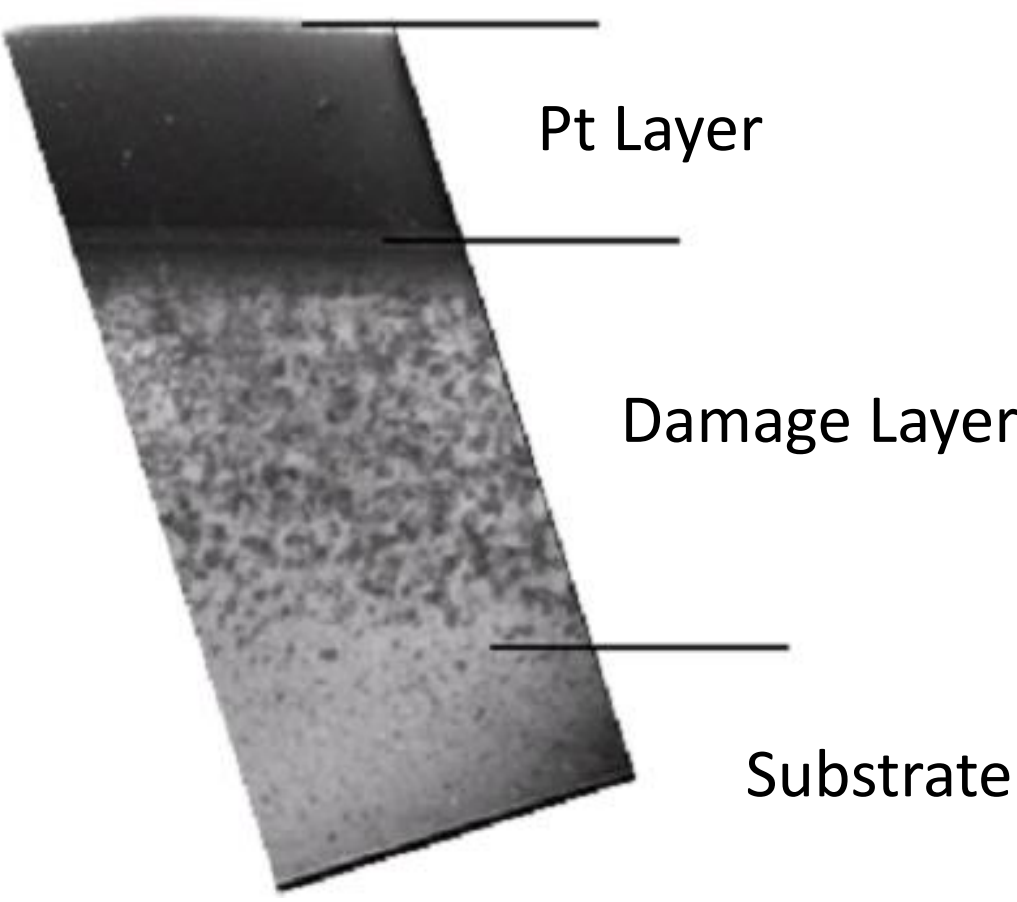

Figure 2 - Cross-sectional TEM image of the damage layer produced in $\mathrm{Fe} 12 \% \mathrm{Cr}$ by $\mathrm{Fe}+$ implantation at $2 \mathrm{MeV}$ and $0.5 \mathrm{MeV}$ (reproduced from ref. [10]). 


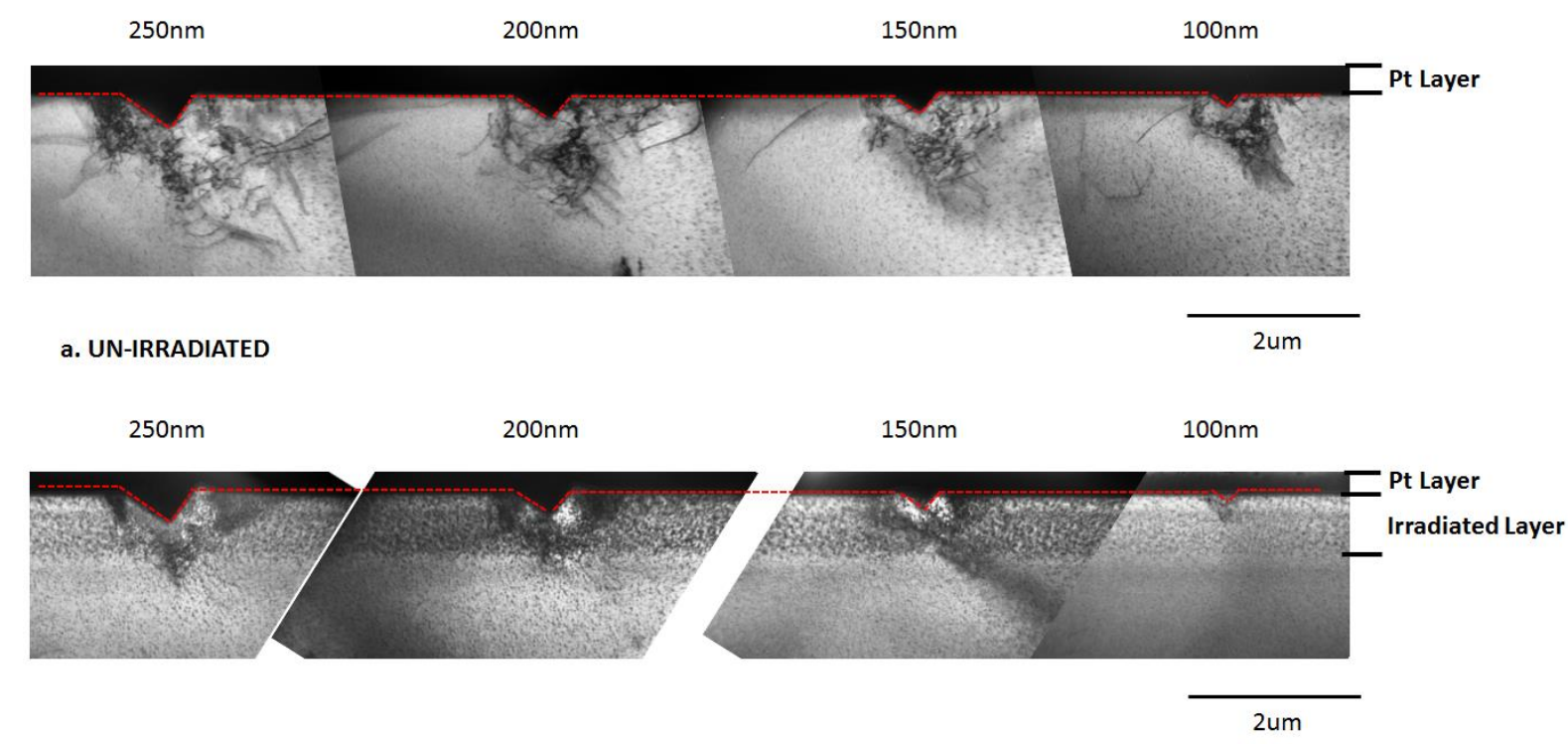

b. IRRADIATED

Figure 3 - Cross-sectional images through the centre of indents with depths from 100 to $250 \mathrm{~nm}$ produced by a cube corner tip in (a) un-irradiated and (b) irradiated regions of the same grain in the Fe12\%Cr sample.

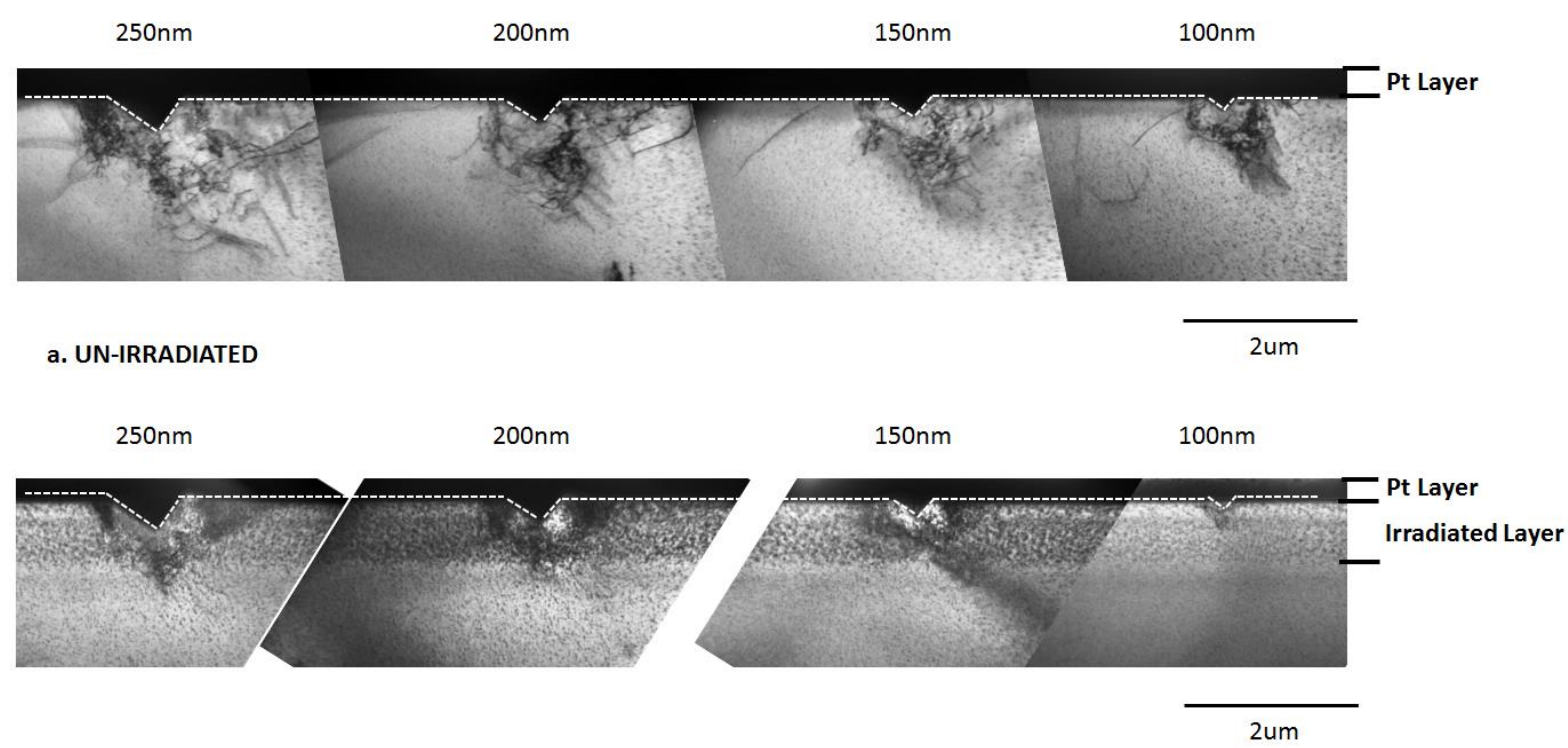

b. IRRADIATED

Figure 3 - Cross-sectional images through the centre of indents with depths from 100 to $250 \mathrm{~nm}$ produced by a cube corner tip in (a) un-irradiated and (b) irradiated regions of the same grain in the Fe12\%Cr sample. 


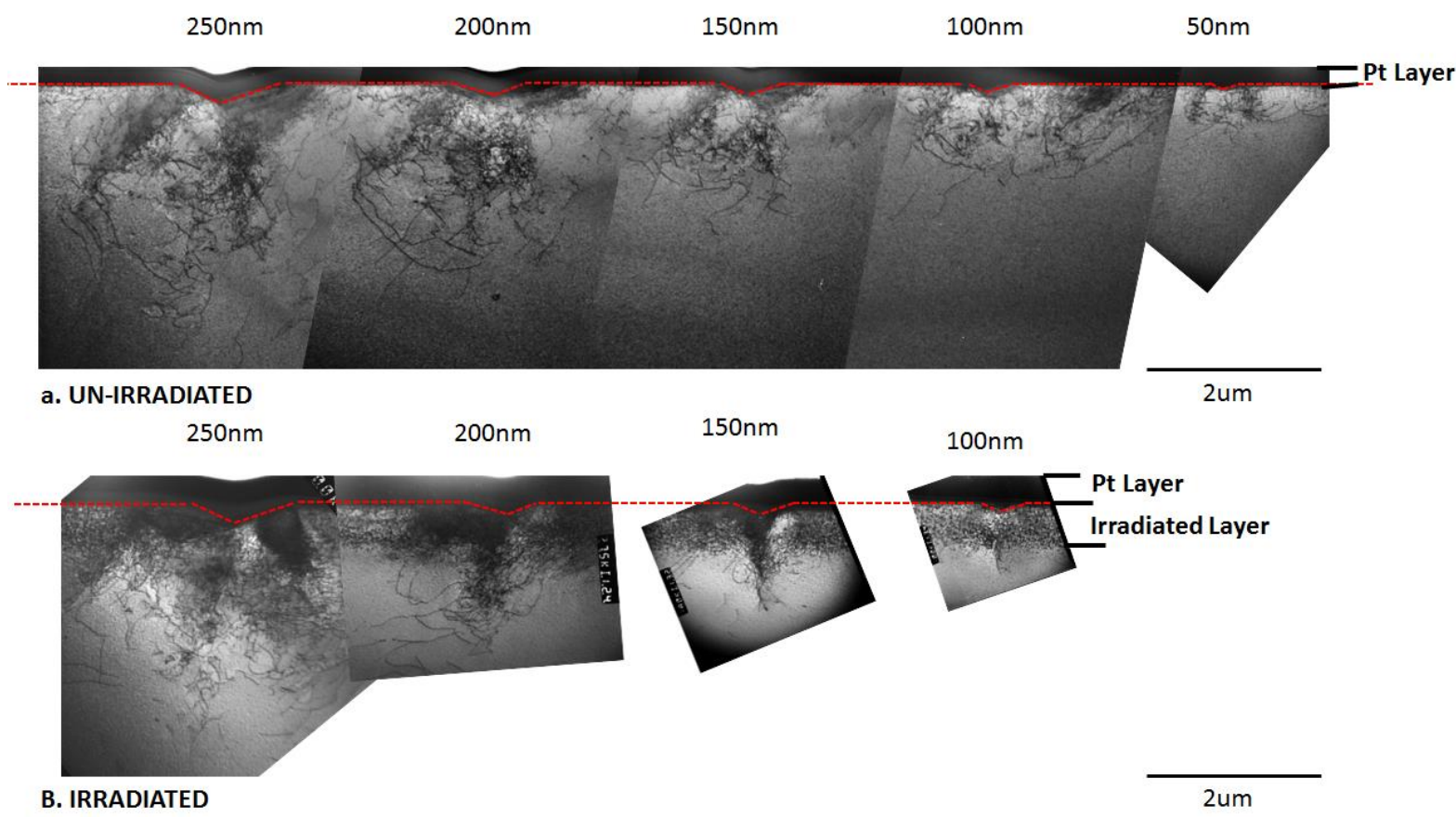

Figure 4 - Cross-sectional images through the centre of indents with depths from 50 to $250 \mathrm{~nm}$ produced by a Berkovich tip in (a) un-irradiated and (b) irradiated regions of the same grain in the Fe12\%Cr sample (reproduced from ref. [10]).

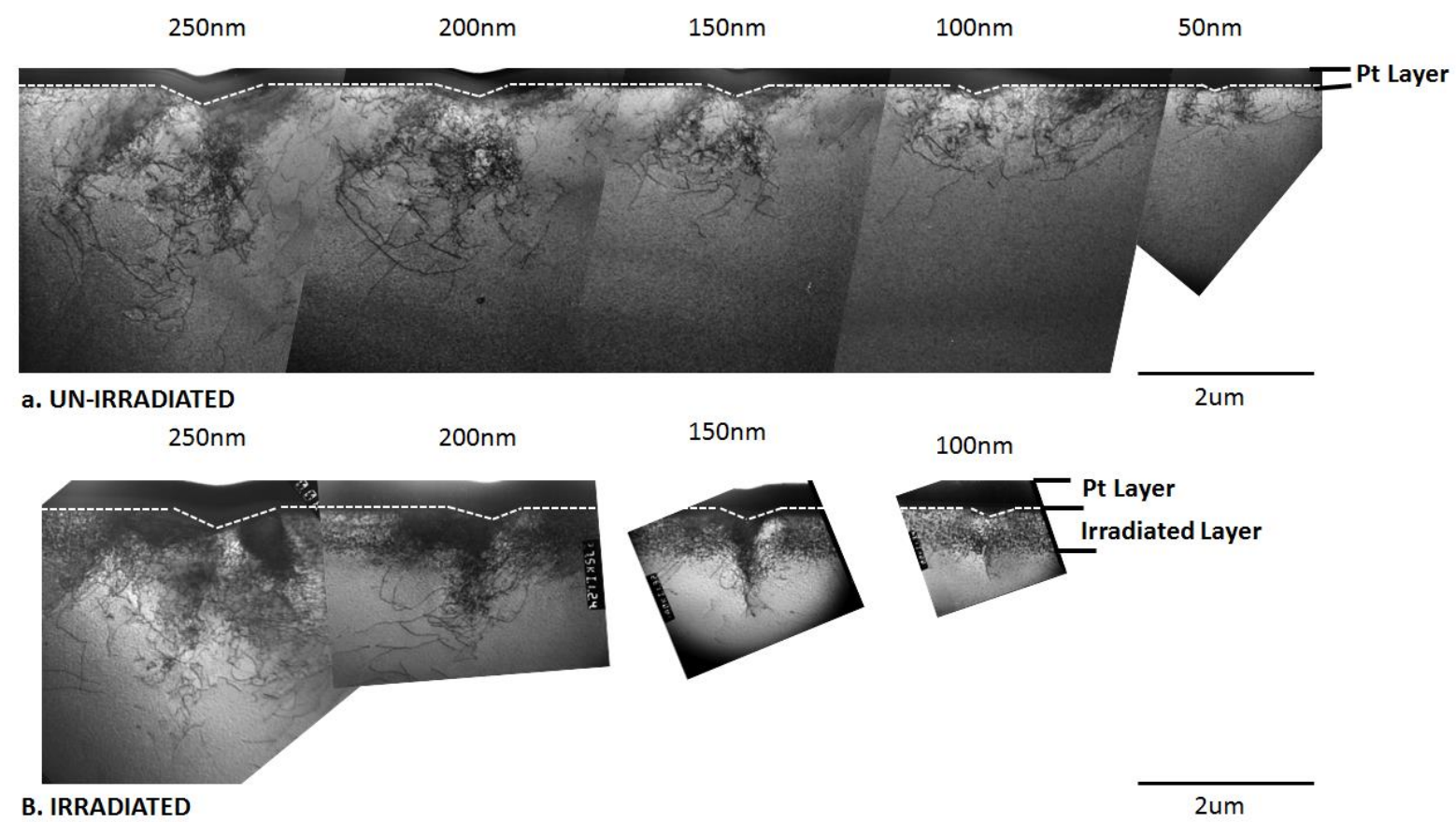

Figure 4 - Cross-sectional images through the centre of indents with depths from 50 to $250 \mathrm{~nm}$ produced by a Berkovich tip in (a) un-irradiated and (b) irradiated regions of the same grain in the Fe12\%Cr sample (reproduced from ref. [10]). 


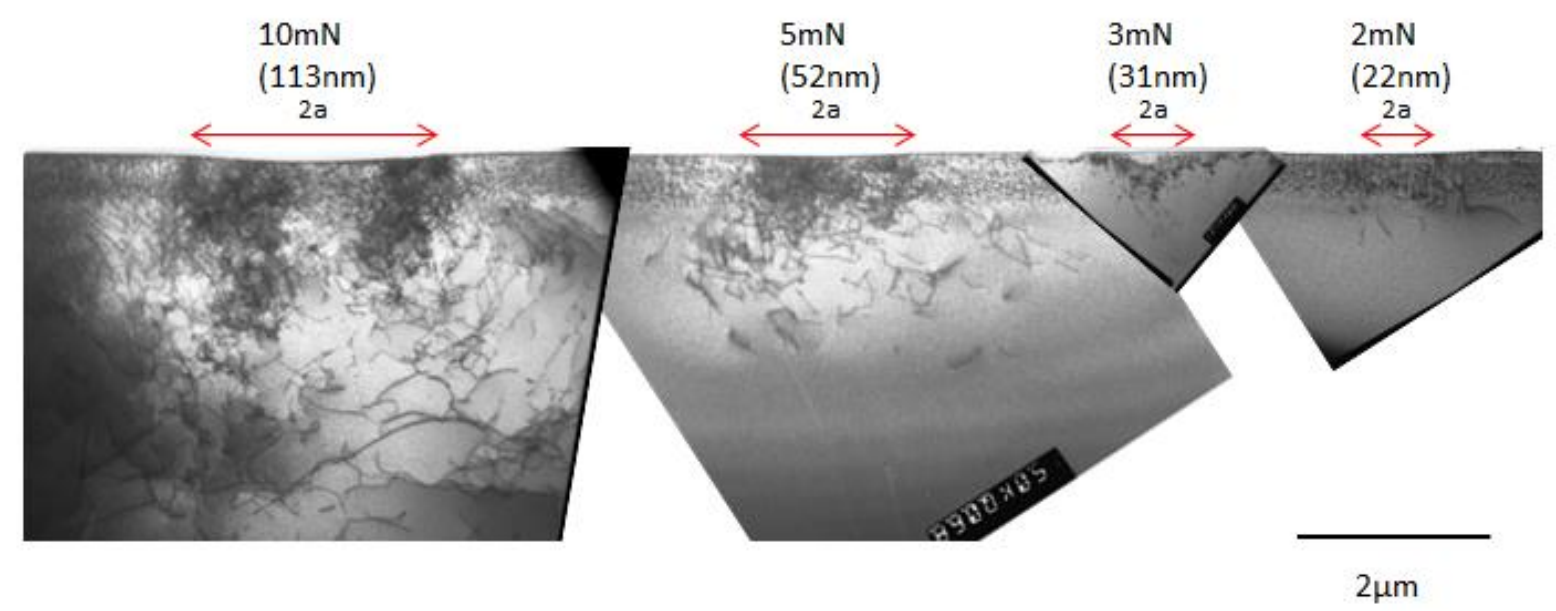

Figure 5 - Cross-sectional images through the centre of spherical indents produced in the irradiated region of the Fe12\%Cr sample. Indents produced with loads from 2 to $10 \mathrm{mN}$ (with corresponding depths from 22 to $113 \mathrm{~nm}$ ) produced by a spherical tip with nominal radius of $10 \mu \mathrm{m}$. The contact area under load has diameter $2 a$ in each case.

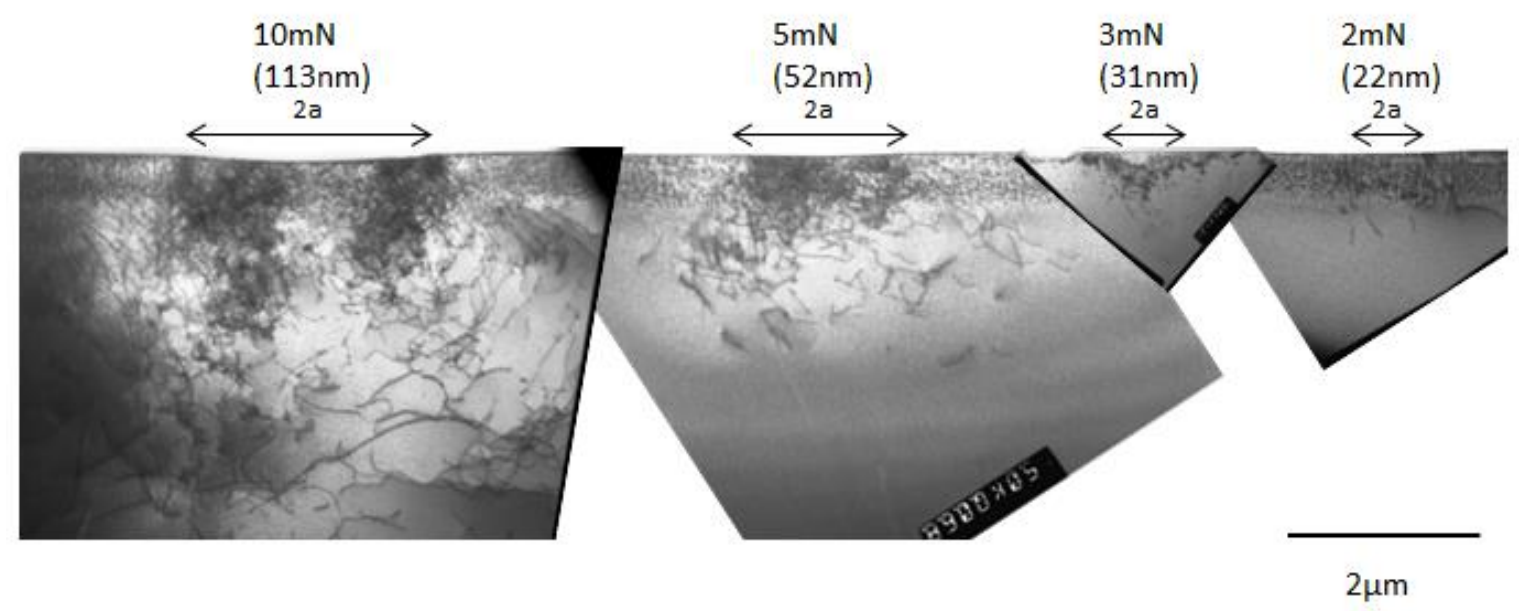

Figure 5 - Cross-sectional images through the centre of spherical indents produced in the irradiated region of the Fe12\% $\mathrm{Cr}$ sample. Indents produced with loads from 2 to $10 \mathrm{mN}$ (with corresponding depths from 22 to $113 \mathrm{~nm}$ ) produced by a spherical tip with nominal radius of $10 \mu \mathrm{m}$. The contact area under load has diameter $2 a$ in each case. 


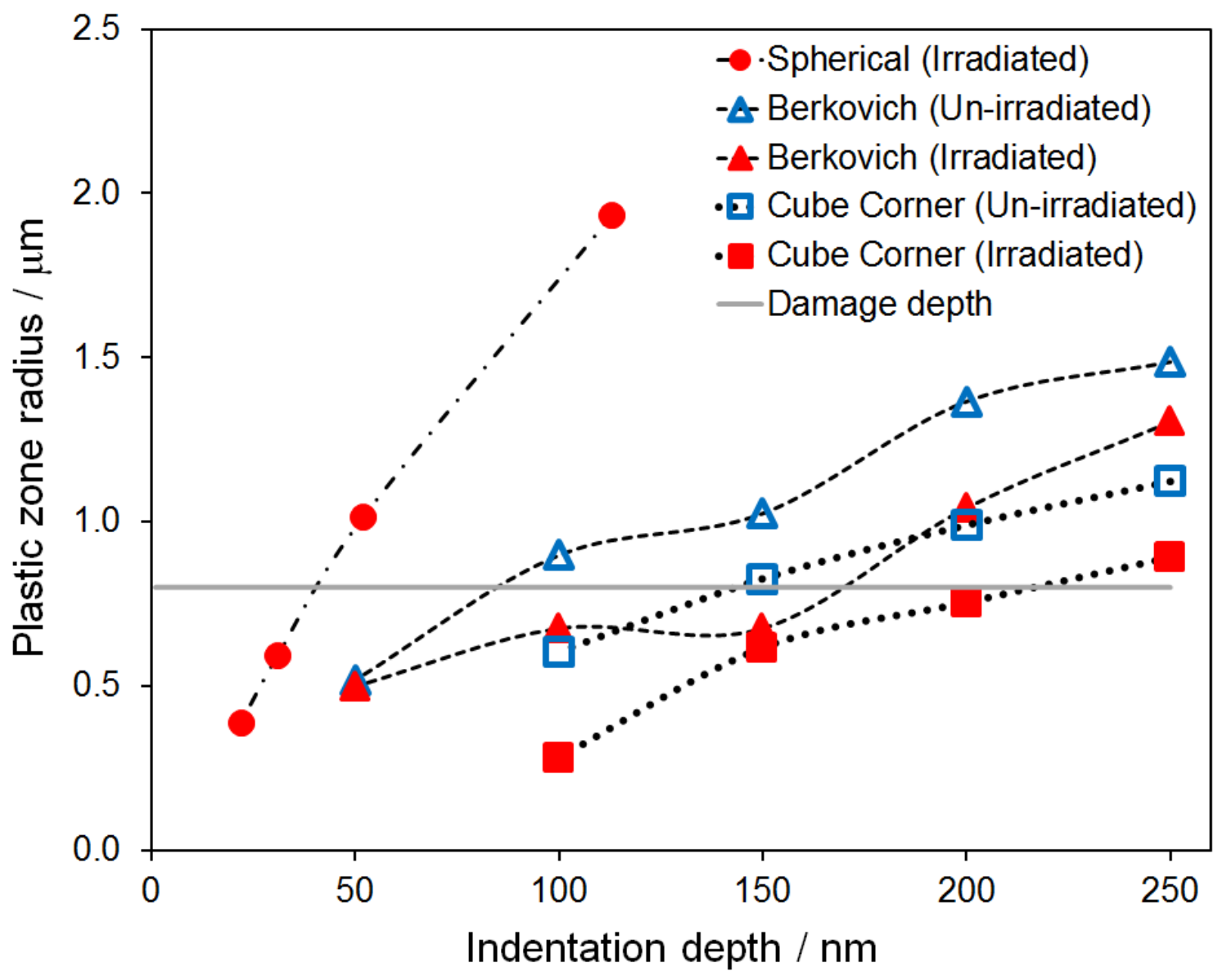

Figure 6 - Plastic zone radius as a function of indentation depth for irradiated (solid red symbols) and un-irradiated (open blue symbols) for the spherical (circle), Berkovich (triangle) and cube corner (square) indenter tip geometries. 


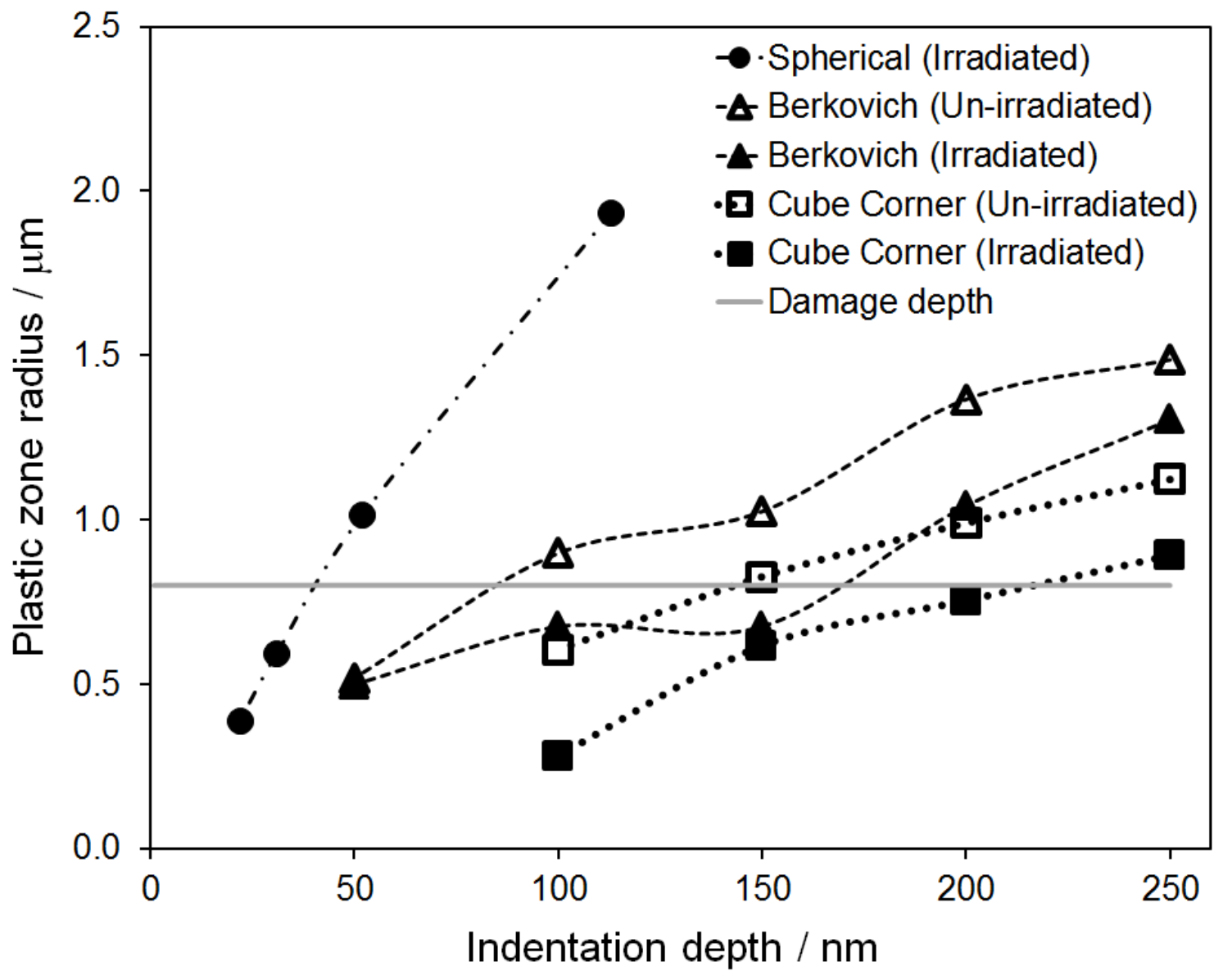

Figure 6 - Plastic zone radius as a function of indentation depth for irradiated (solid symbols) and un-irradiated (open symbols) for the spherical (circle), Berkovich (triangle) and cube corner (square) indenter tip geometries. 


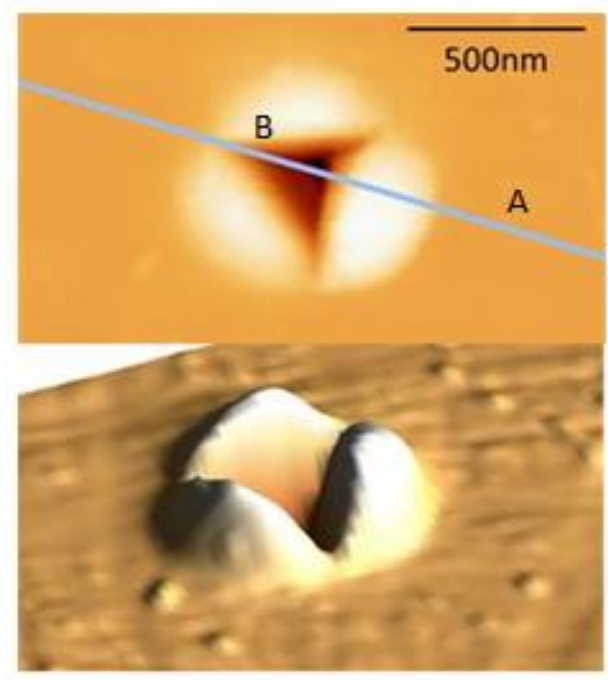

a.

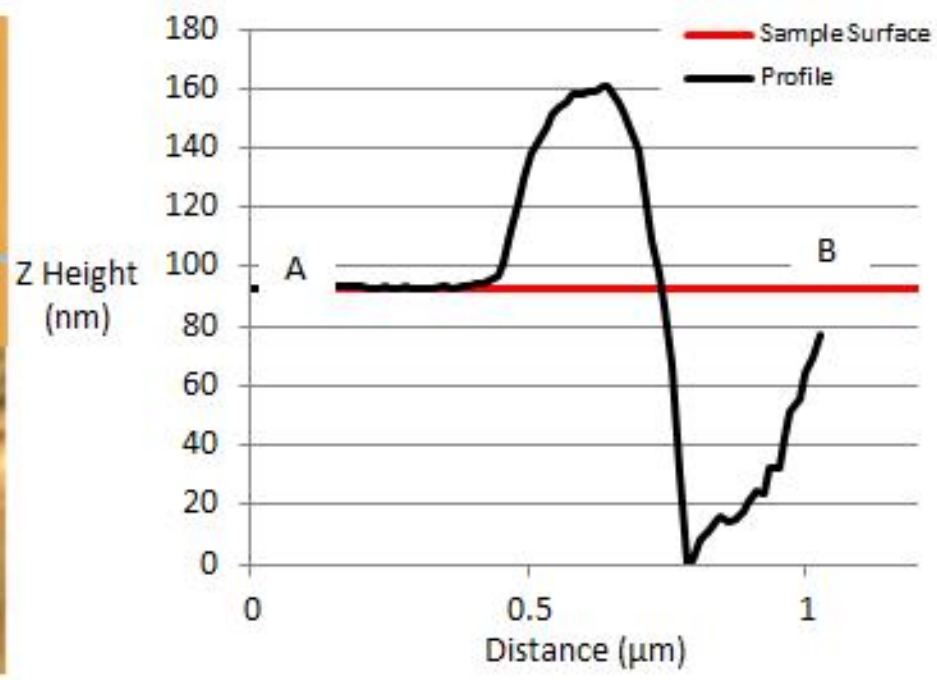

b.

Figure 7-AFM tomography of a 200nm depth indent with a cube corner tip in an irradiated region of Fe12\% Cr shown in 2D with line profile marker and 3D (a) and height data from line profile (b).

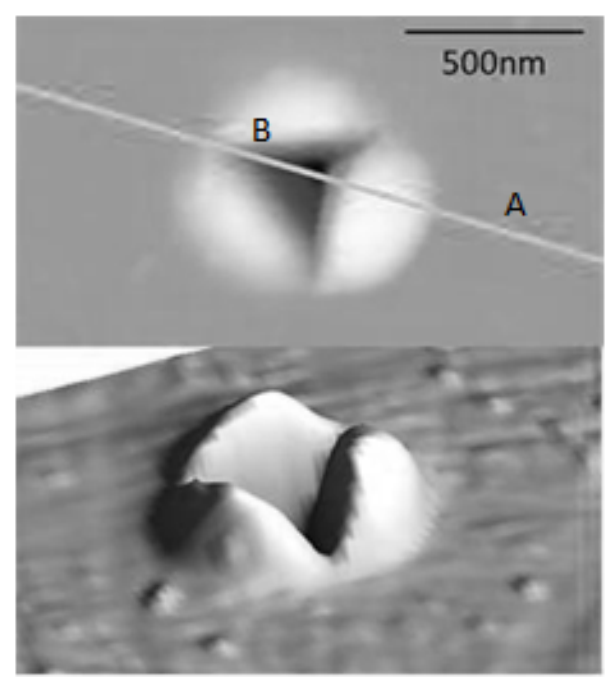

a.

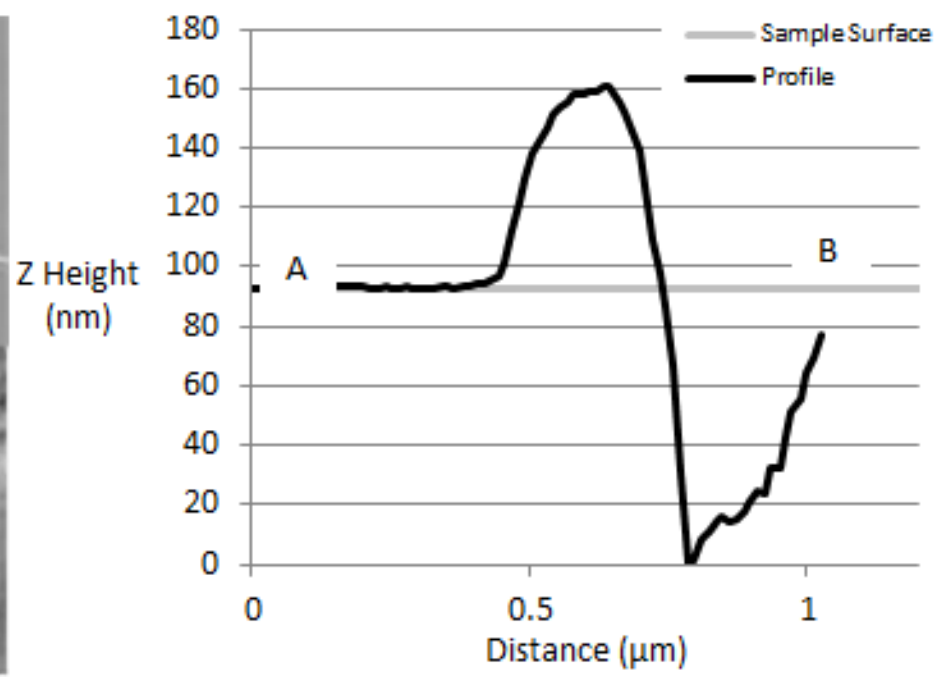

b.

Figure 7 - AFM tomography of a 200nm depth indent with a cube corner tip in an irradiated region of Fe12\% Cr shown in 2D with line profile marker and 3D (a) and height data from line profile (b). 
UN-IRRADIATED
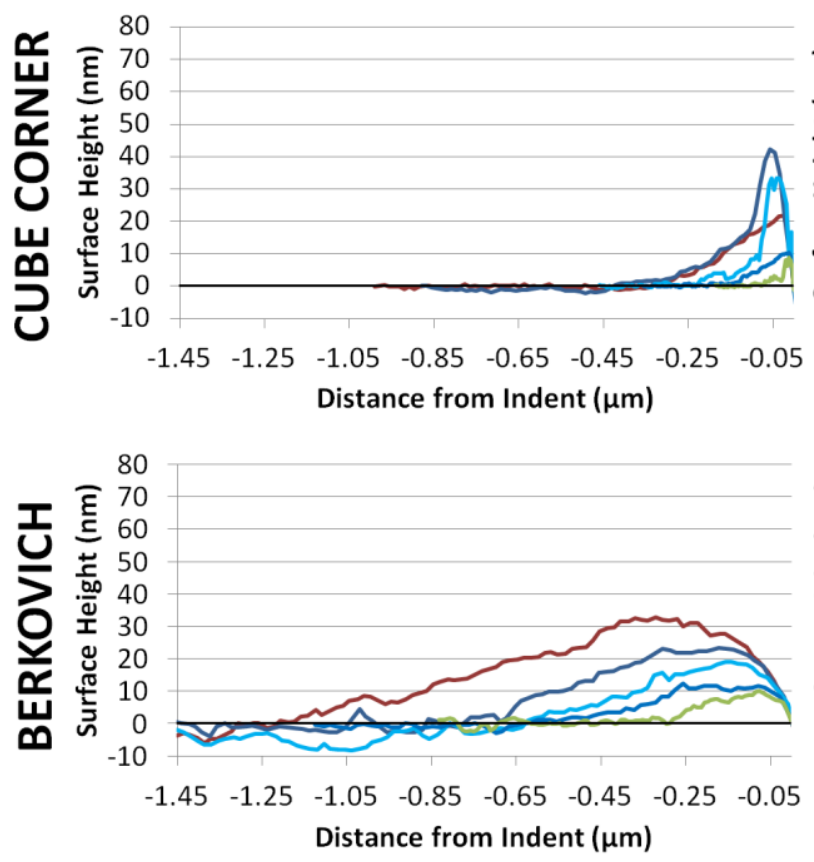

IRRADIATED
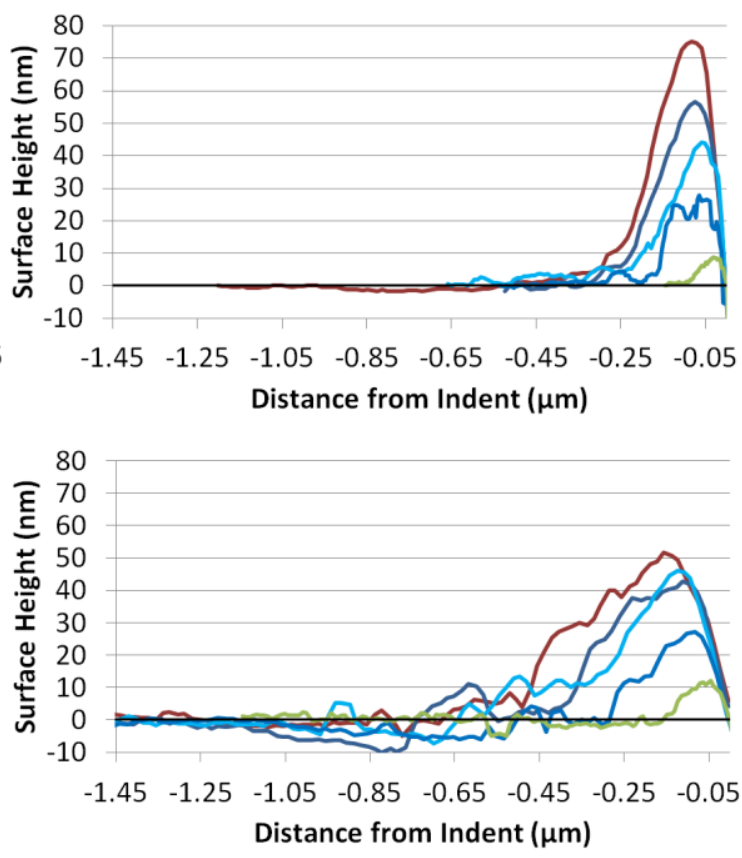

$-250 \mathrm{~nm}-200 \mathrm{~nm}-150 \mathrm{~nm}-100 \mathrm{~nm}-50 \mathrm{~nm}$

Figure 8 - AFM line profile through pile-up lobes of indentations of depths from 50 to $250 \mathrm{~nm}$ produced by both Berkovich and cube corner tips. Profiles corrected for surface height and alignment with flat surface of indenter tip on the right in each case. 
UN-IRRADIATED
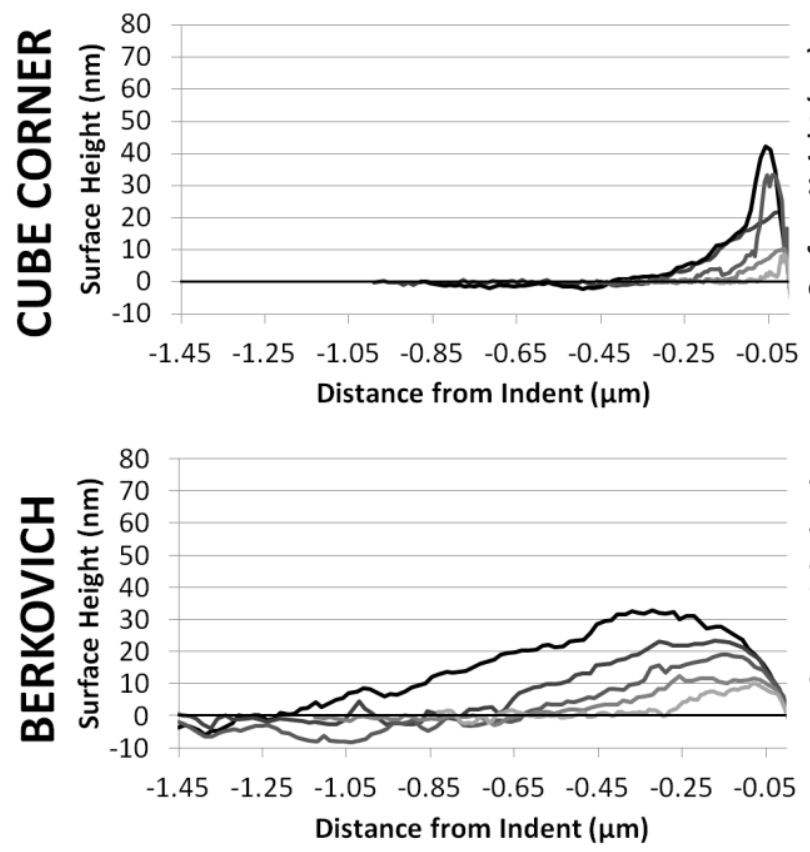

Indent ( $\mu \mathrm{m})$
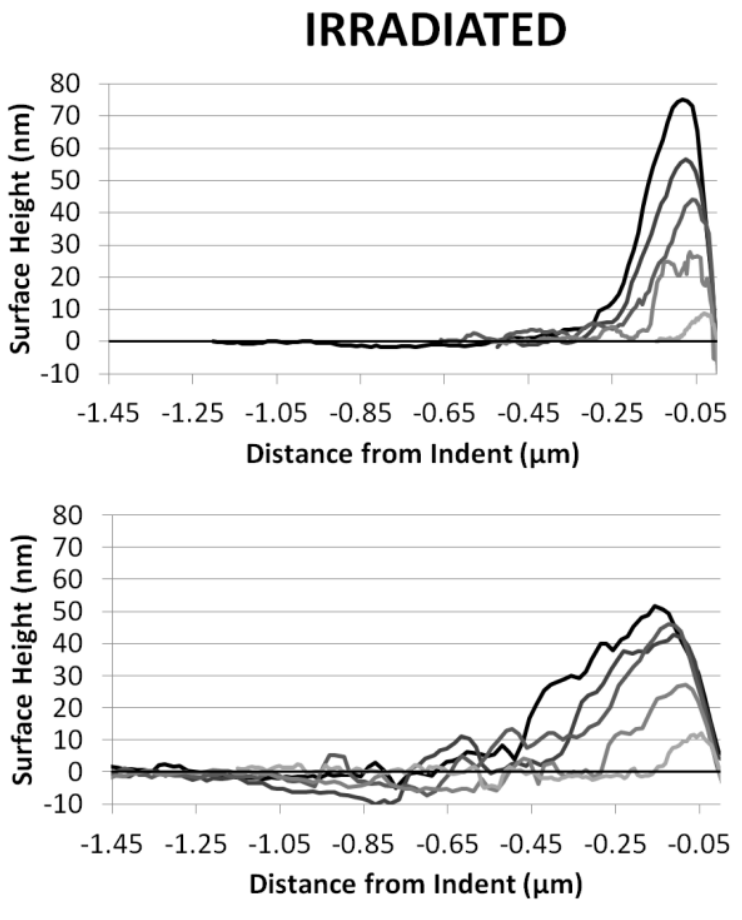

Figure 8 - AFM line profile through pile-up lobes of indentations of depths from 50 to $250 \mathrm{~nm}$ produced by both Berkovich and cube corner tips. Profiles corrected for surface height and alignment with flat surface of indenter tip on the right in each case. 


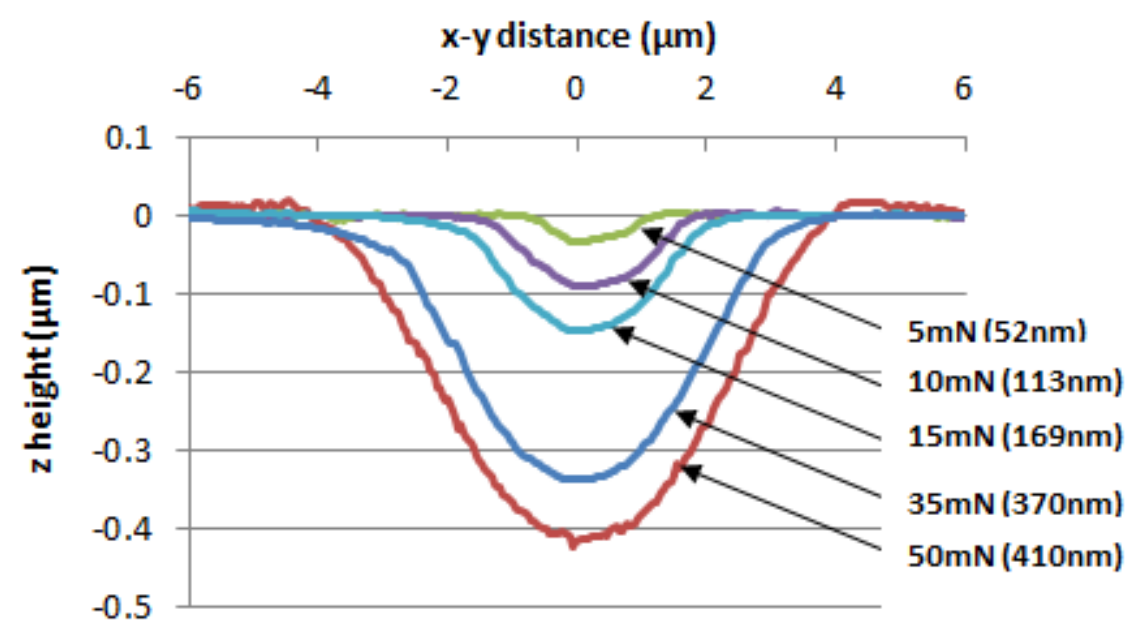

Figure 9 - AFM line traces through deepest pixel of indentation for indents produced by a spherical tip (nominal radius $10 \mu \mathrm{m}$ ) at loads of $50 \mathrm{mN}, 35 \mathrm{mN}, 15 \mathrm{mN}, 10 \mathrm{mN}$ and $5 \mathrm{mN}$ (corresponding indentation depth in brackets).

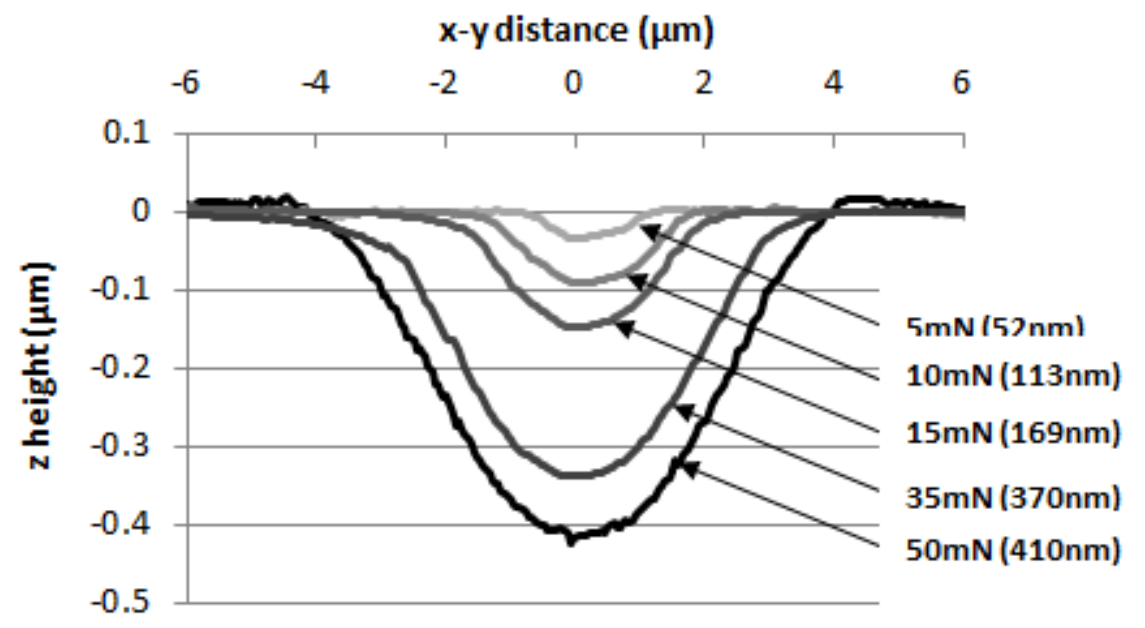

Figure 9 - AFM line traces through deepest pixel of indentation for indents produced by a spherical tip (nominal radius $10 \mu \mathrm{m}$ ) at loads of $50 \mathrm{mN}, 35 \mathrm{mN}, 15 \mathrm{mN}, 10 \mathrm{mN}$ and $5 \mathrm{mN}$ (corresponding indentation depth in brackets). 


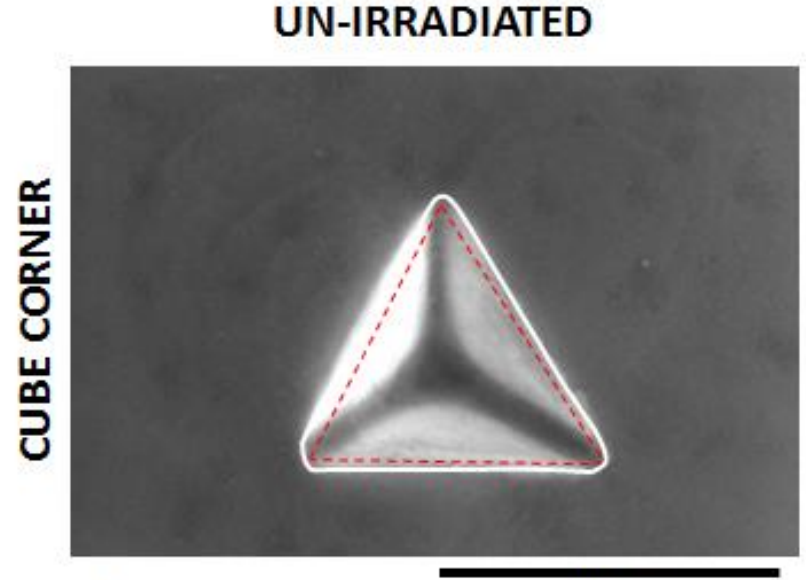

a.

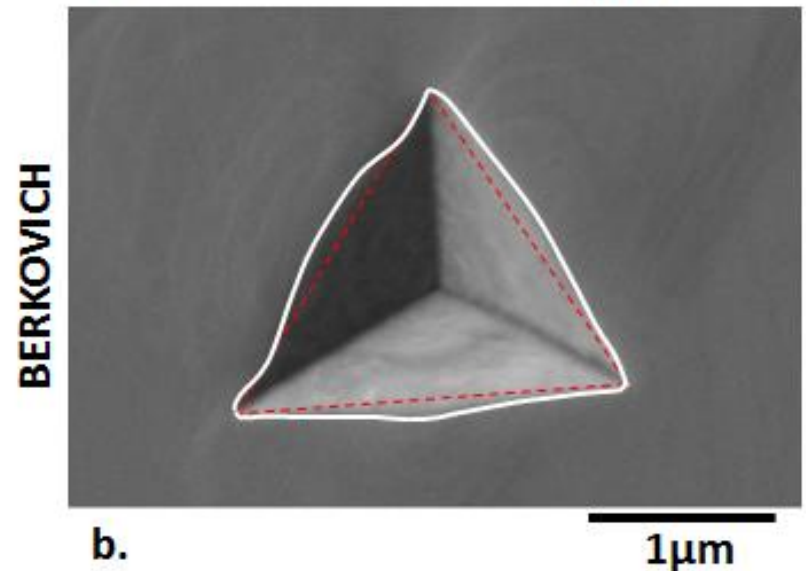

IRRADIATED

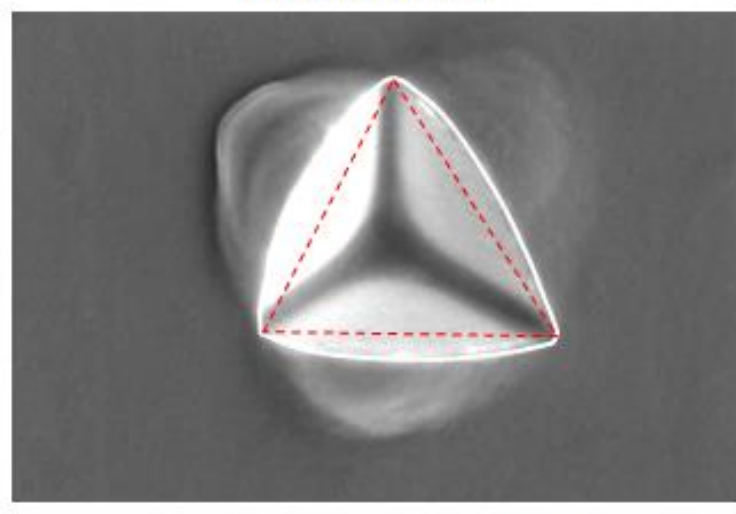

$1 \mu \mathrm{m}$

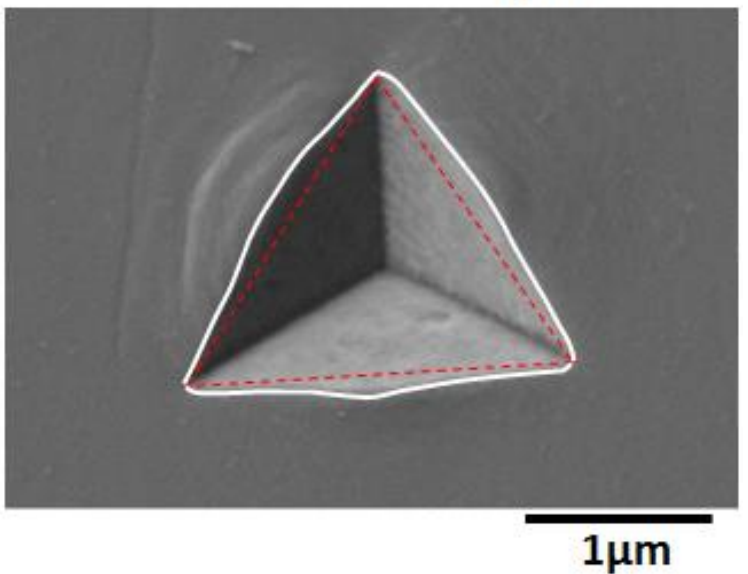

Figure 10 - SEM images showing corner-to-corner measurement $\left(A_{c c}\right)$ of contact area (red dashed) and actual $\left(A_{\text {actual }}\right)$ contact area (white solid) for $250 \mathrm{~nm}$ deep cube corner (a) and Berkovich (b) indents in irradiated and un-irradiated regions of the same grain. 


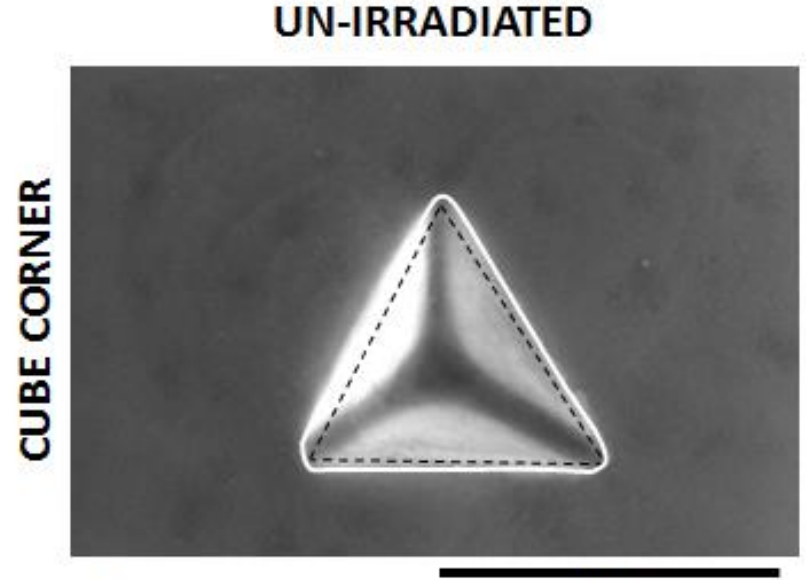

a.

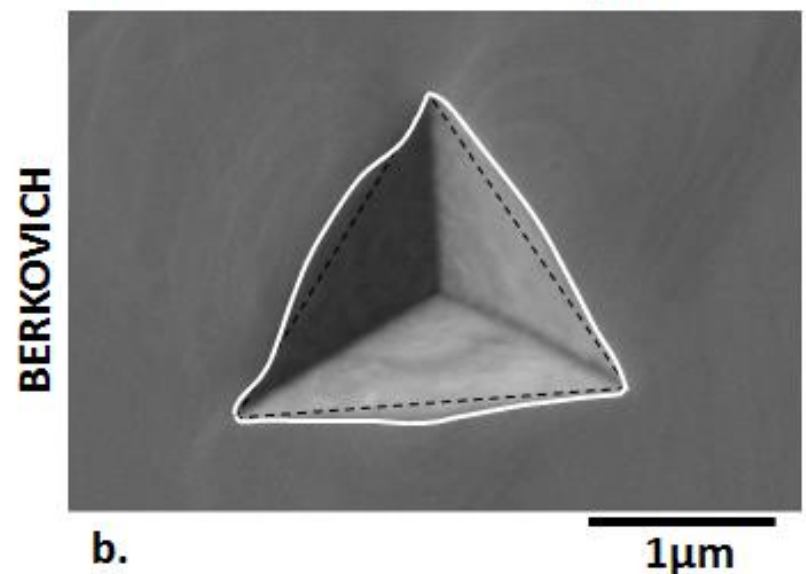

IRRADIATED

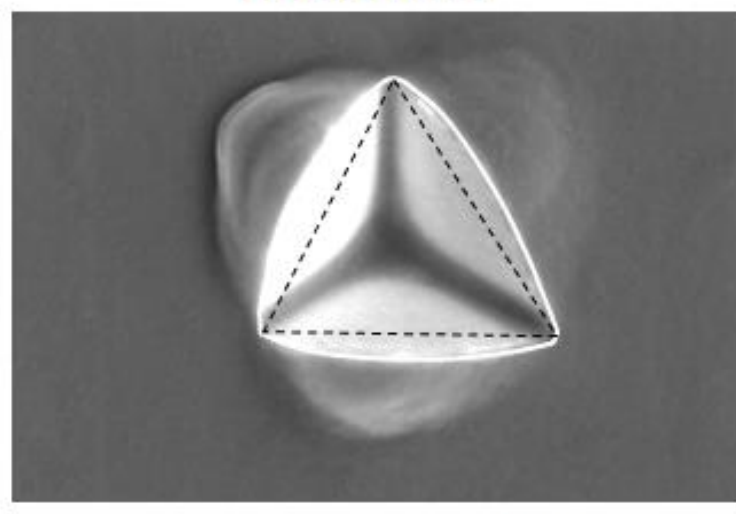

$1 \mu \mathrm{m}$

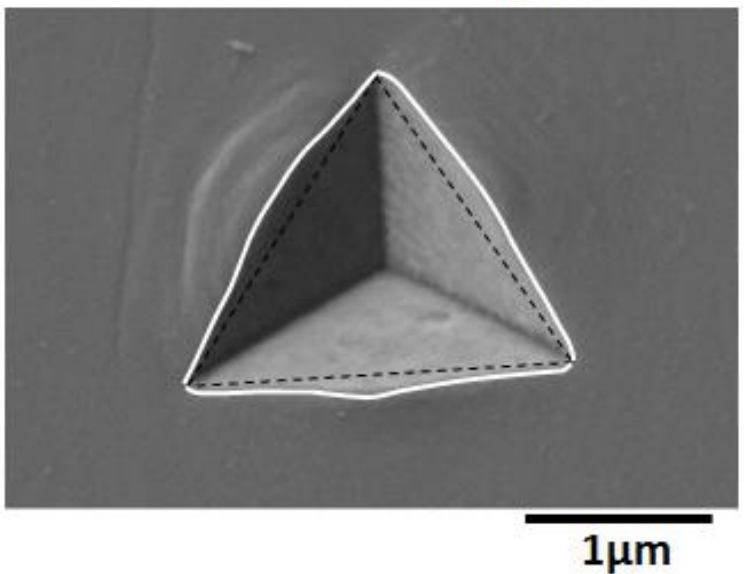

Figure 10 - SEM images showing corner-to-corner measurement $\left(A_{c c}\right)$ of contact area (black dashed) and actual ( $\left.A_{\text {actual }}\right)$ contact area (white solid) for $250 \mathrm{~nm}$ deep cube corner (a) and Berkovich (b) indents in irradiated and un-irradiated regions of the same grain. 

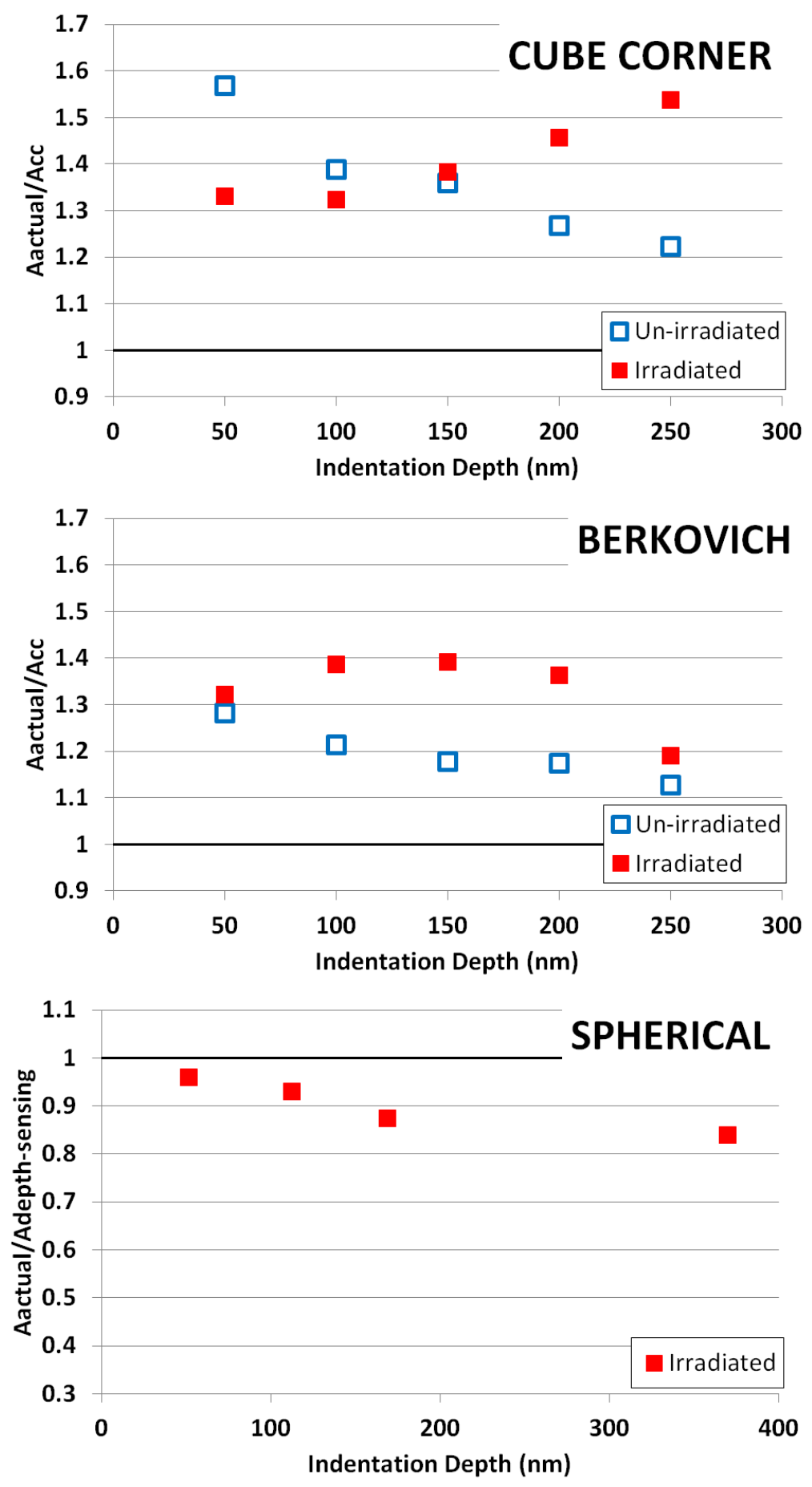

Figure 11 - Ratio of actual contact area $\left(A_{\text {actual }}\right)$ to the corner-to-corner $\left(A_{c c}\right)$ measurement $\left(A_{\text {actual }} / A_{c c}\right)$ of indentations produced by the cube corner and Berkovich tips in the irradiated (red solid) and un-irradiated (blue open) material. $A_{c c}$ is replaced with the depth-sensing calculation ( $\left.A_{\text {actual }} / A_{\text {depth-sensing }}\right)$ for indentations produced by the spherical tip. Data are plotted against indentation depth $(\mathrm{nm})$ for all tips. 

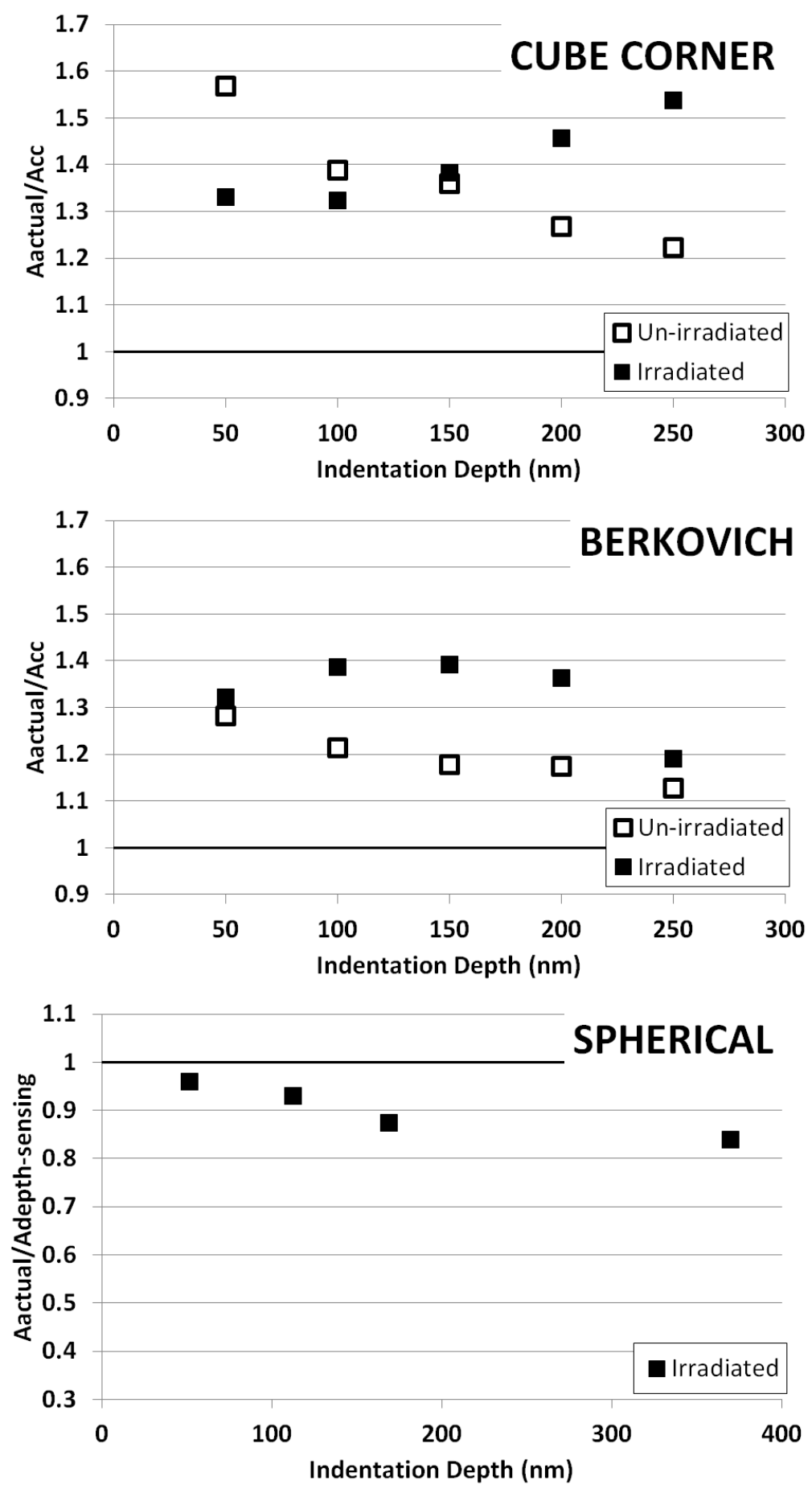

Figure 11 - Ratio of actual contact area ( $\left.A_{\text {actual }}\right)$ to the corner-to-corner $\left(A_{c c}\right)$ measurement ( $A_{\text {actual }} / A_{c c}$ ) of indentations produced by the cube corner and Berkovich tips in the irradiated (solid) and un-irradiated (open) material. $A_{c c}$ is replaced with the depth-sensing calculation $\left(A_{\text {actual }} / A_{\text {depth- }}\right.$ sensing) for indentations produced by the spherical tip. Data are plotted against indentation depth $(n m)$ for all tips. 

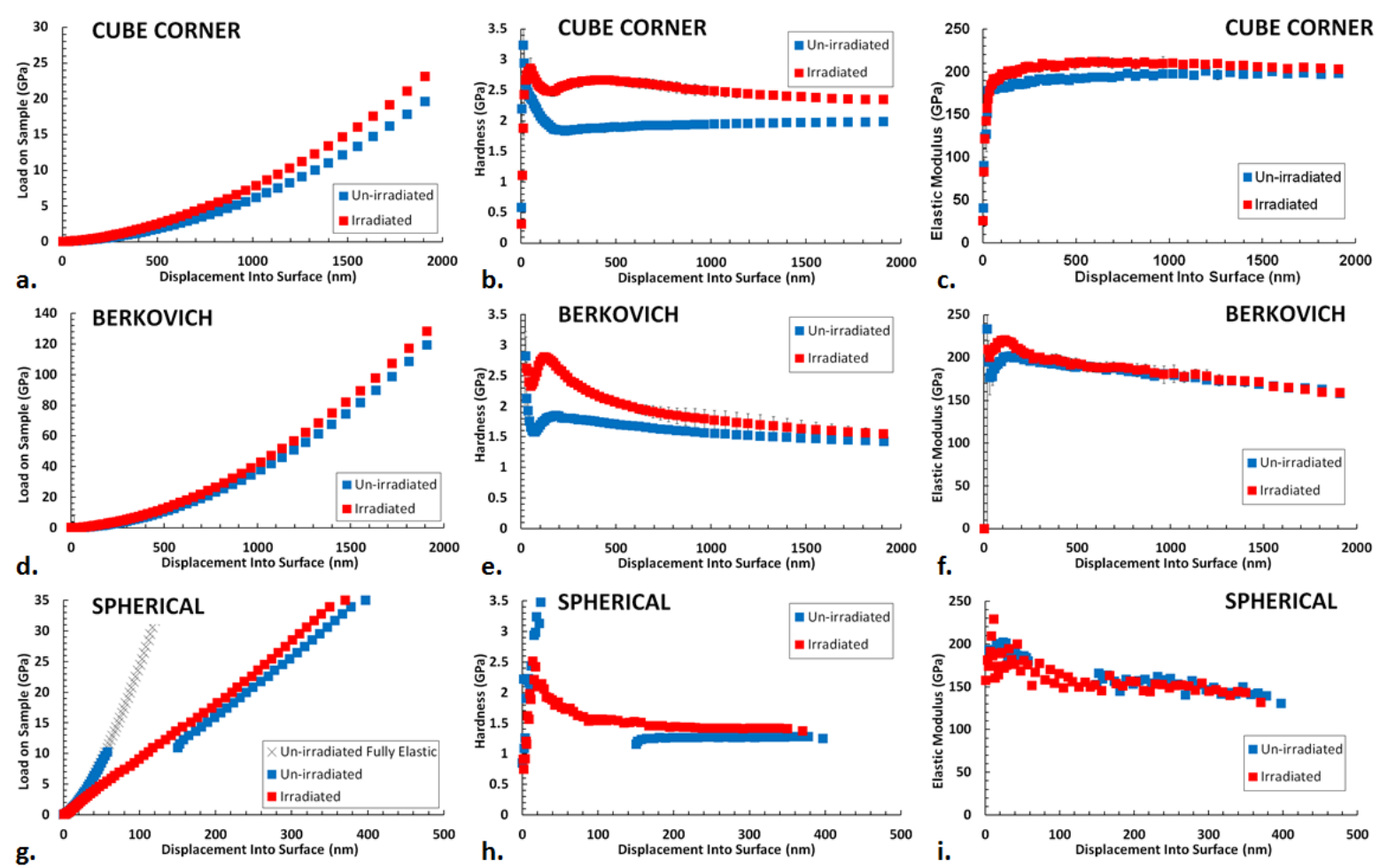

Figure 12 - Load on sample, hardness and elastic modulus indentation data for un-irradiated (blue) and irradiated (red) regions of $\mathrm{Fe} 12 \% \mathrm{Cr}$, produced by cube corner, Berkovich and spherical tips.
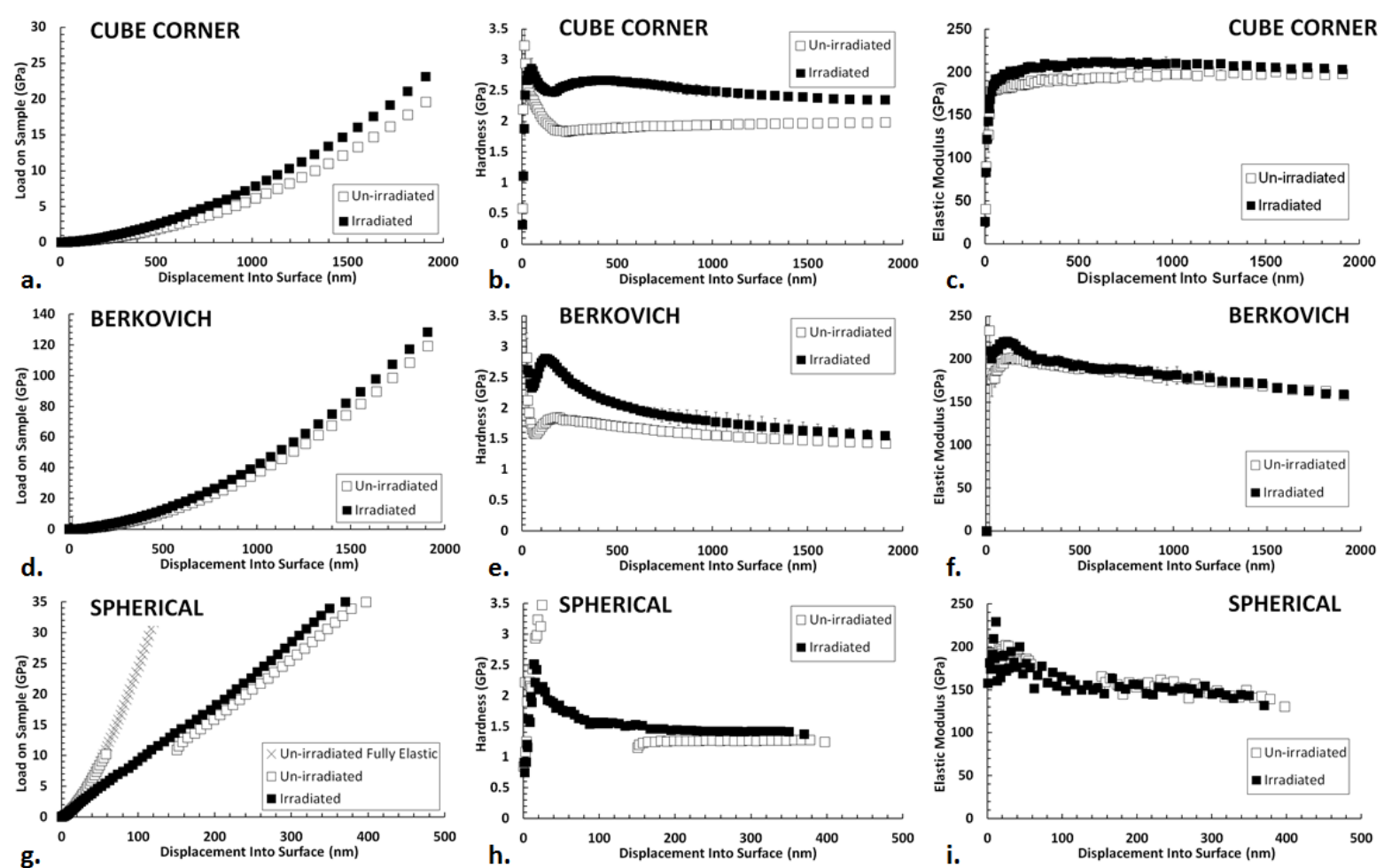

Figure 12 - Load on sample, hardness and elastic modulus indentation data for un-irradiated (open symbols) and irradiated (solid symbols) regions of $\mathrm{Fe} 12 \% \mathrm{Cr}$, produced by cube corner, Berkovich and spherical tips. 

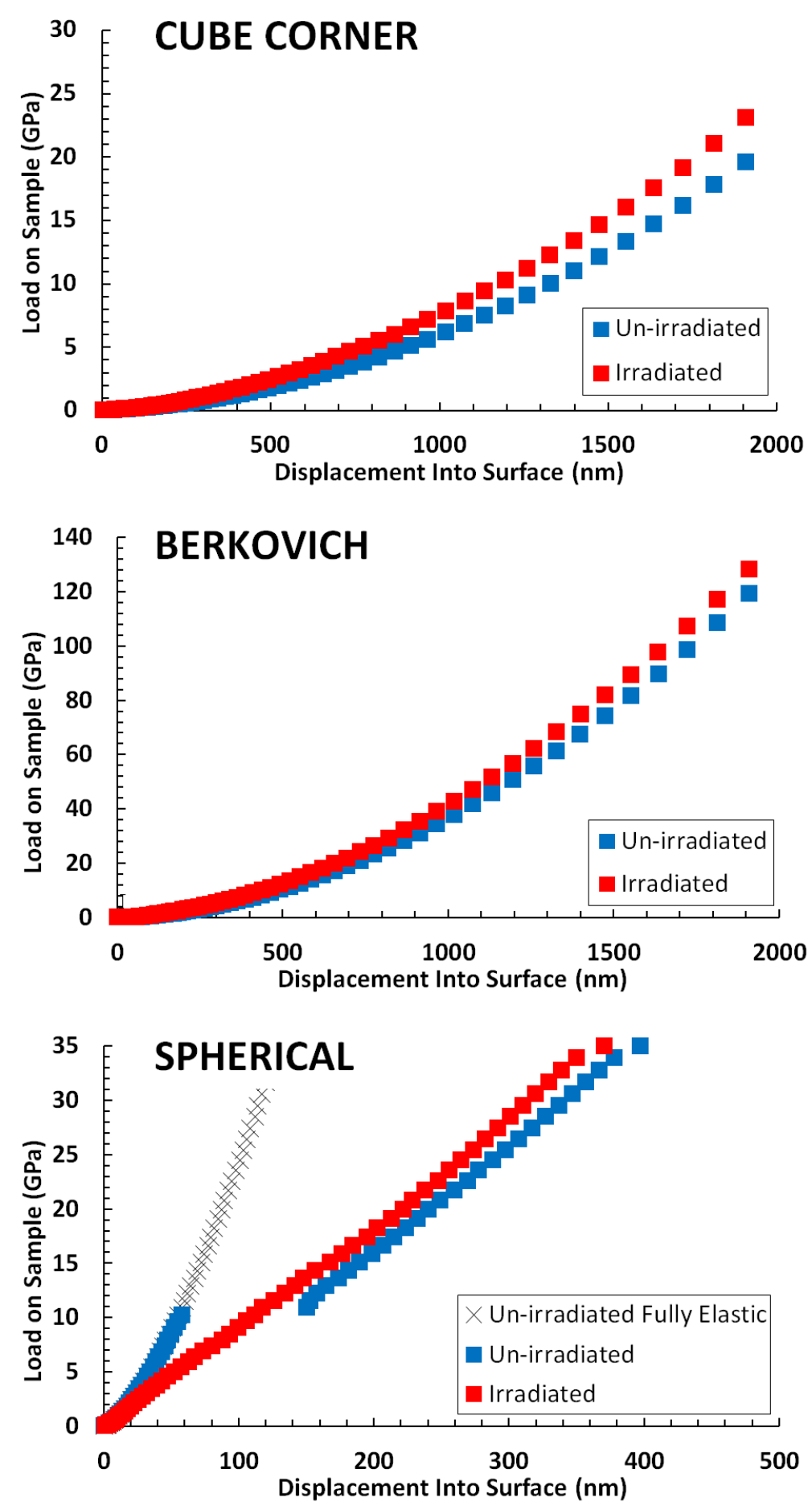

Alternative A- Figure 12 - Load on sample indentation data for un-irradiated (blue symbols) and irradiated (red symbols) regions of $\mathrm{Fe} 12 \% \mathrm{Cr}$, produced by cube corner, Berkovich and spherical tips. 

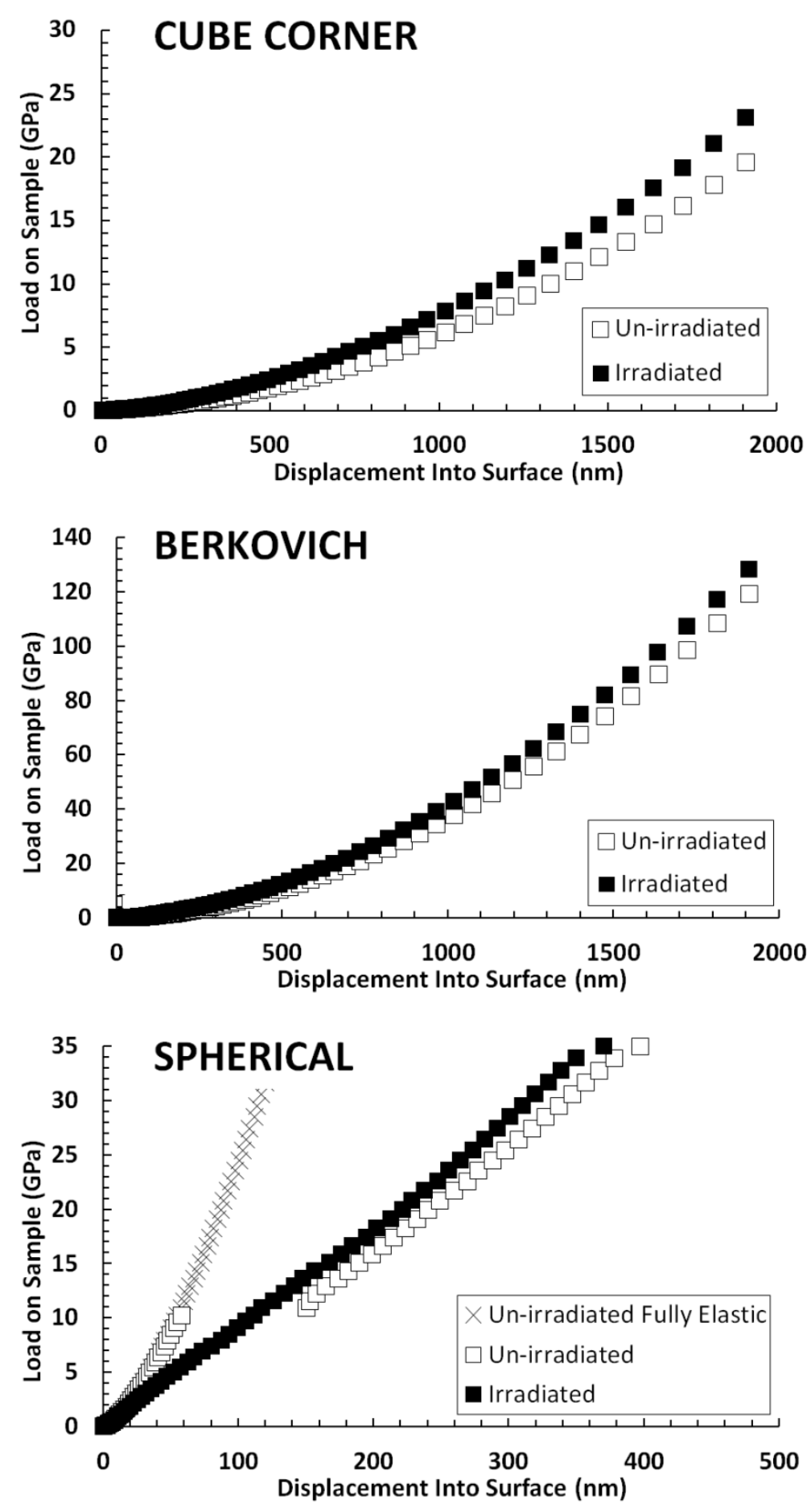

Alternative A- Figure 12 - Load on sample indentation data for un-irradiated (open symbols) and irradiated (solid symbols) regions of $\mathrm{Fe} 12 \% \mathrm{Cr}$, produced by cube corner, Berkovich and spherical tips. 

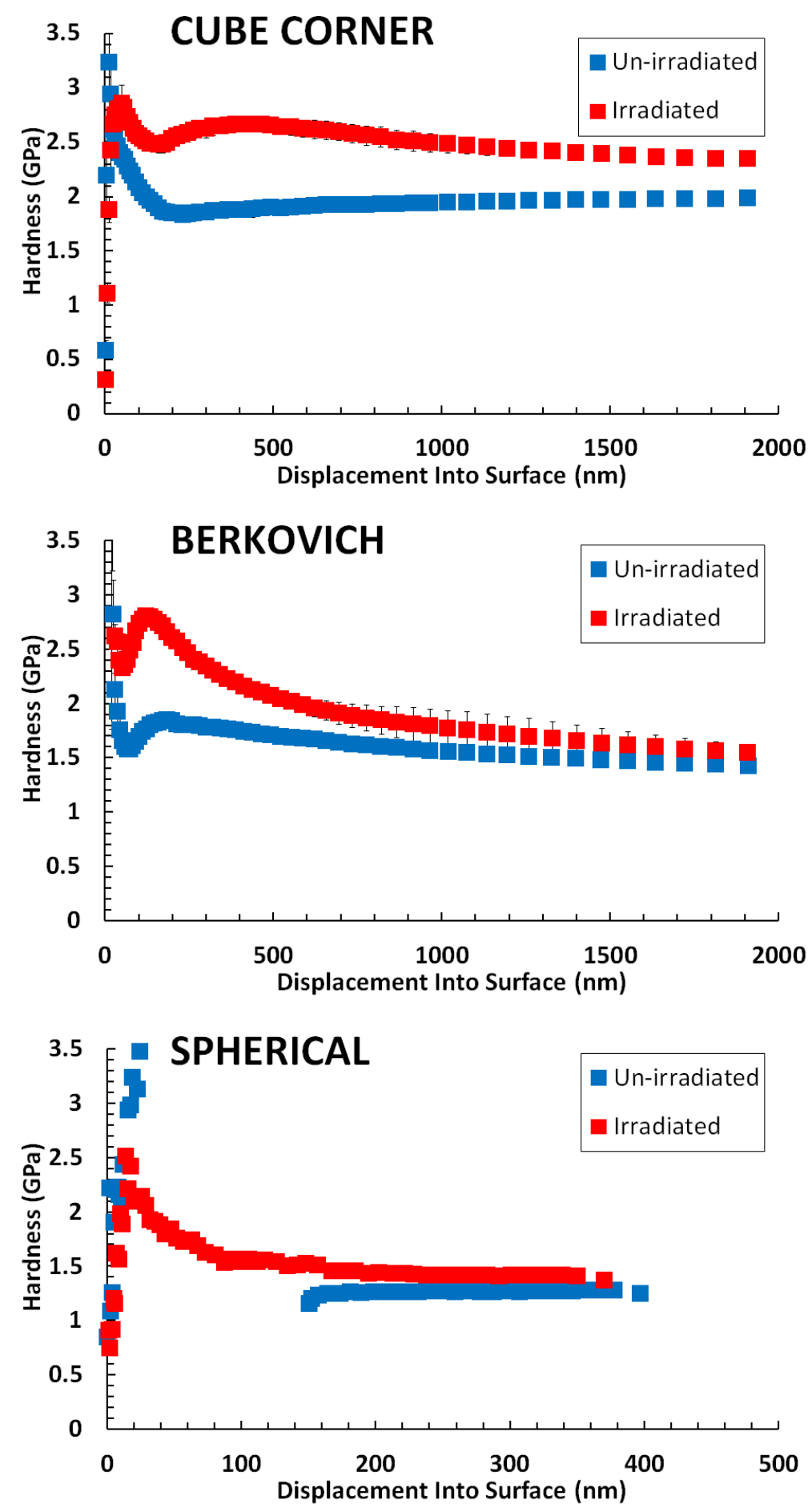

Alternative B- Figure 12 - Hardness data for un-irradiated (blue symbols) and irradiated (red symbols) regions of $\mathrm{Fe} 12 \% \mathrm{Cr}$, produced by cube corner, Berkovich and spherical tips. 

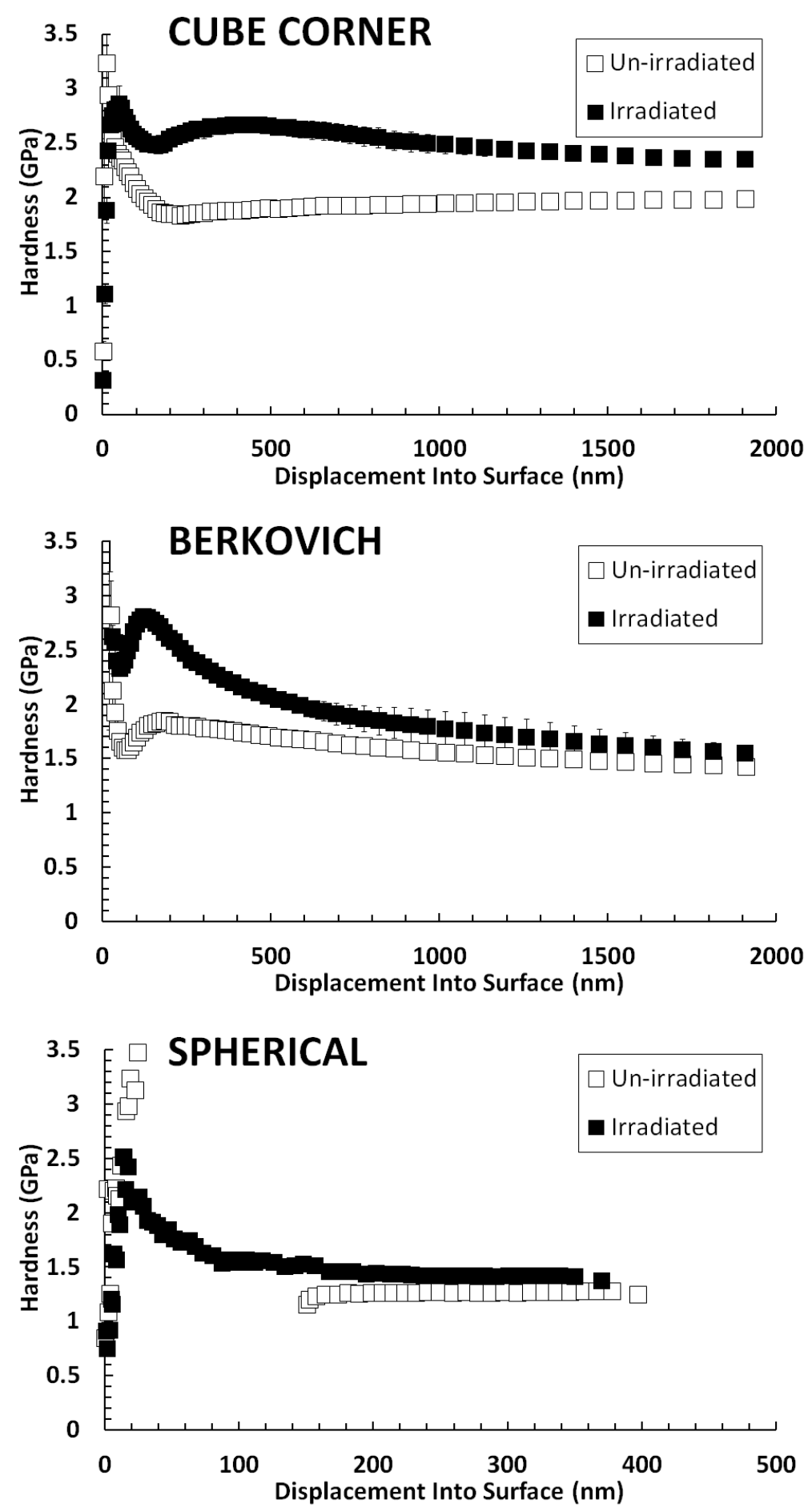

Alternative B- Figure 12 - Hardness data for un-irradiated (open symbols) and irradiated (solid symbols) regions of $\mathrm{Fe} 12 \% \mathrm{Cr}$, produced by cube corner, Berkovich and spherical tips. 

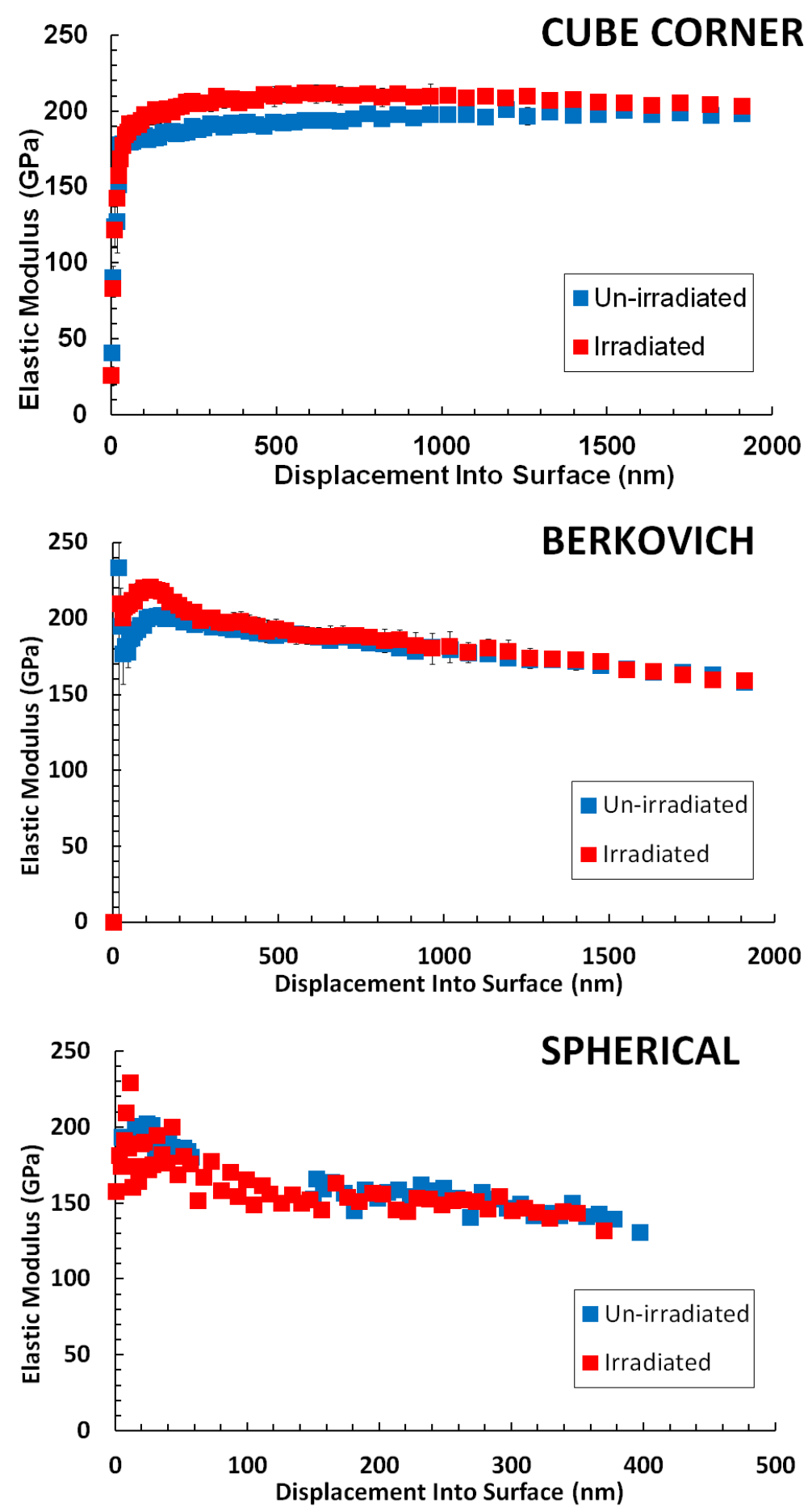

Alternative C- Figure 12 - Elastic modulus data for un-irradiated (blue symbols) and irradiated (red symbols) regions of $\mathrm{Fe} 12 \% \mathrm{Cr}$, produced by cube corner, Berkovich and spherical tips. 

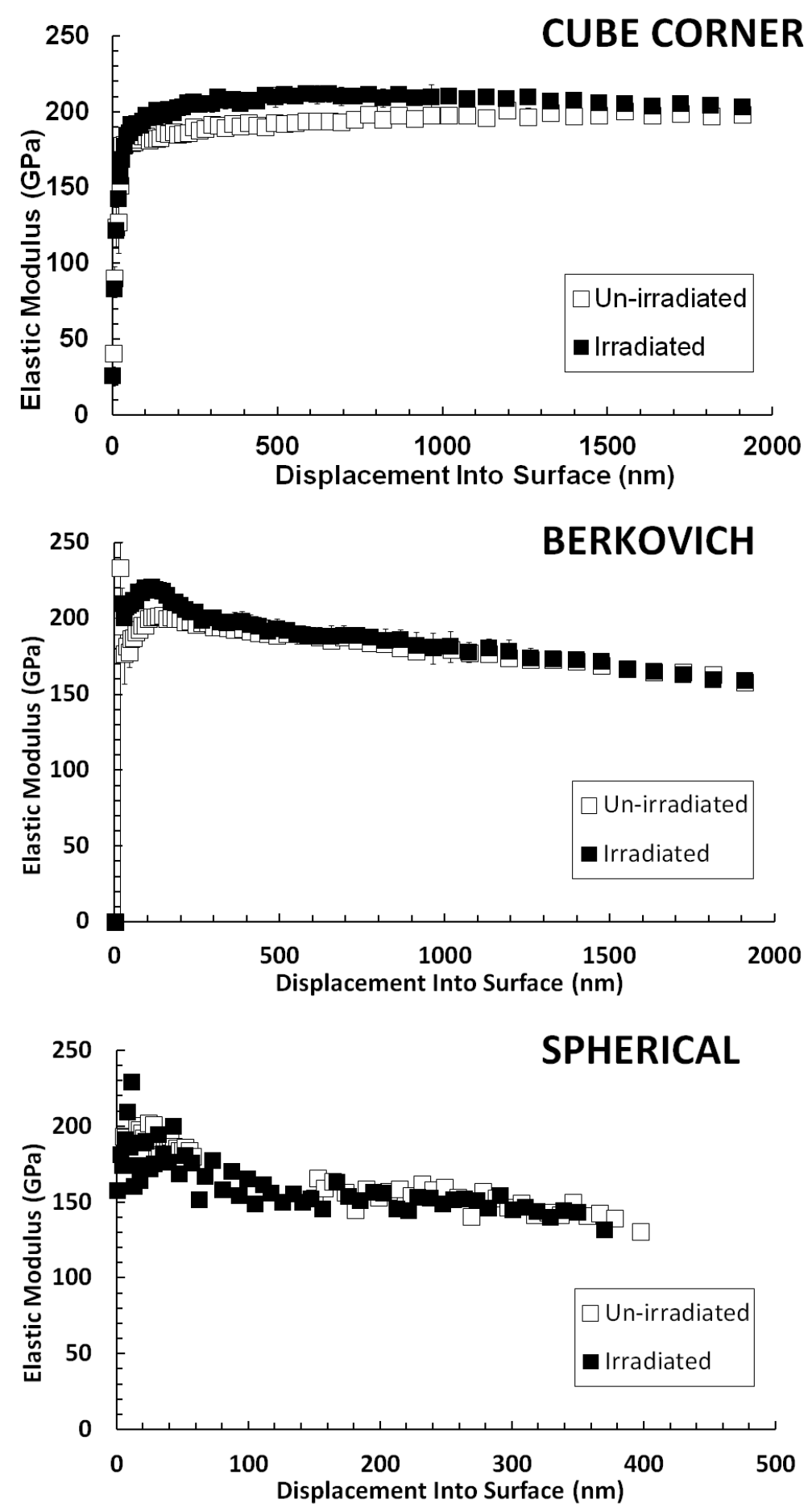

Alternative C- Figure 12 - Hardness data for un-irradiated (open symbols) and irradiated (solid symbols) regions of $\mathrm{Fe} 12 \% \mathrm{Cr}$, produced by cube corner, Berkovich and spherical tips. 

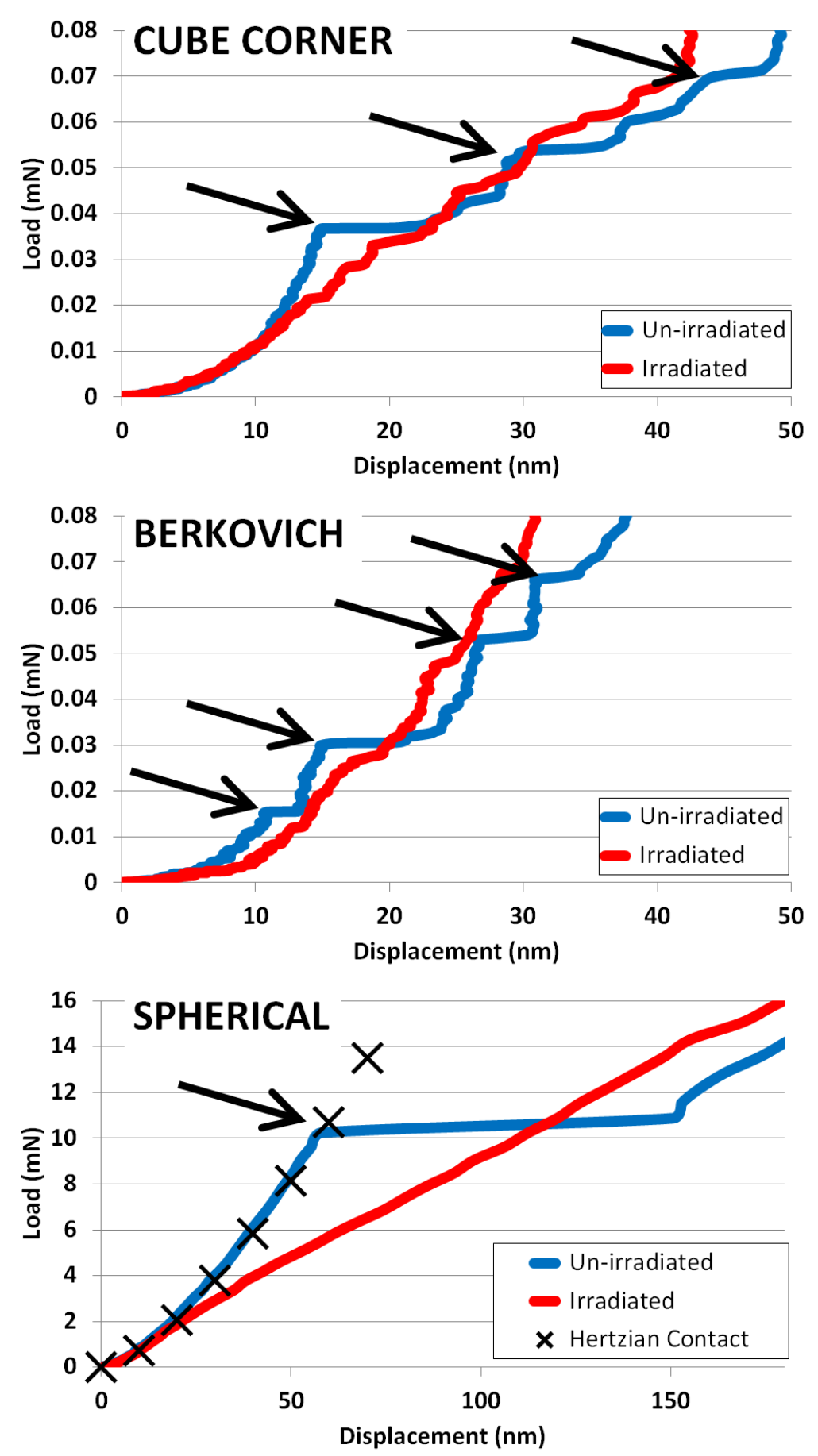

Figure 13 - Typical load-depth data produced with indentation with the cube corner, Berkovich and spherical tips into un-irradiated (blue line) and irradiated (red line) material; black arrows indicate pop-in events in the un-irradiated material. 

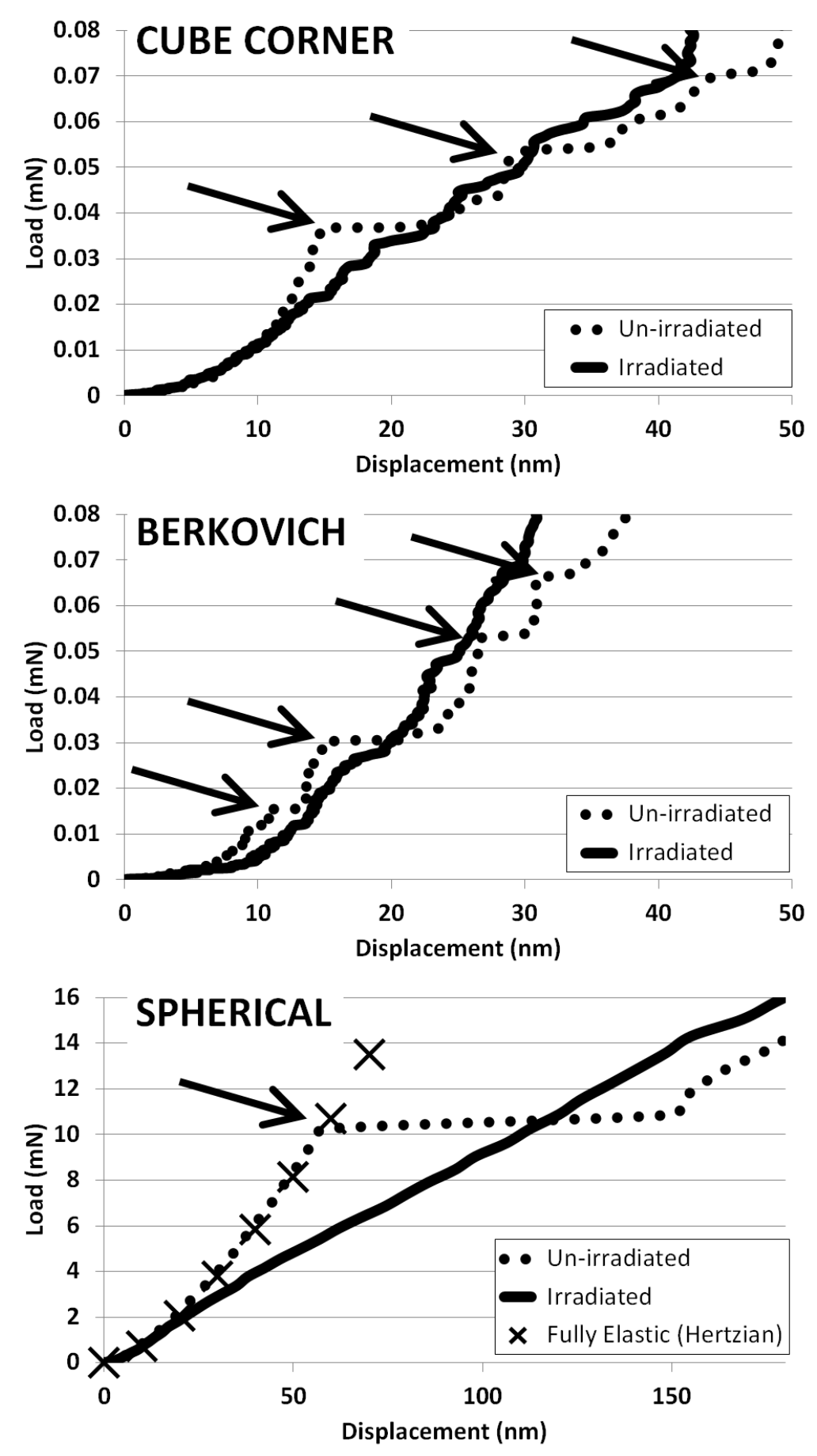

Figure 13 - Typical load-depth data produced with indentation with the cube corner, Berkovich and spherical tips into un-irradiated (dashed line) and irradiated (solid line) material; black arrows indicate pop-in events in the un-irradiated material. 

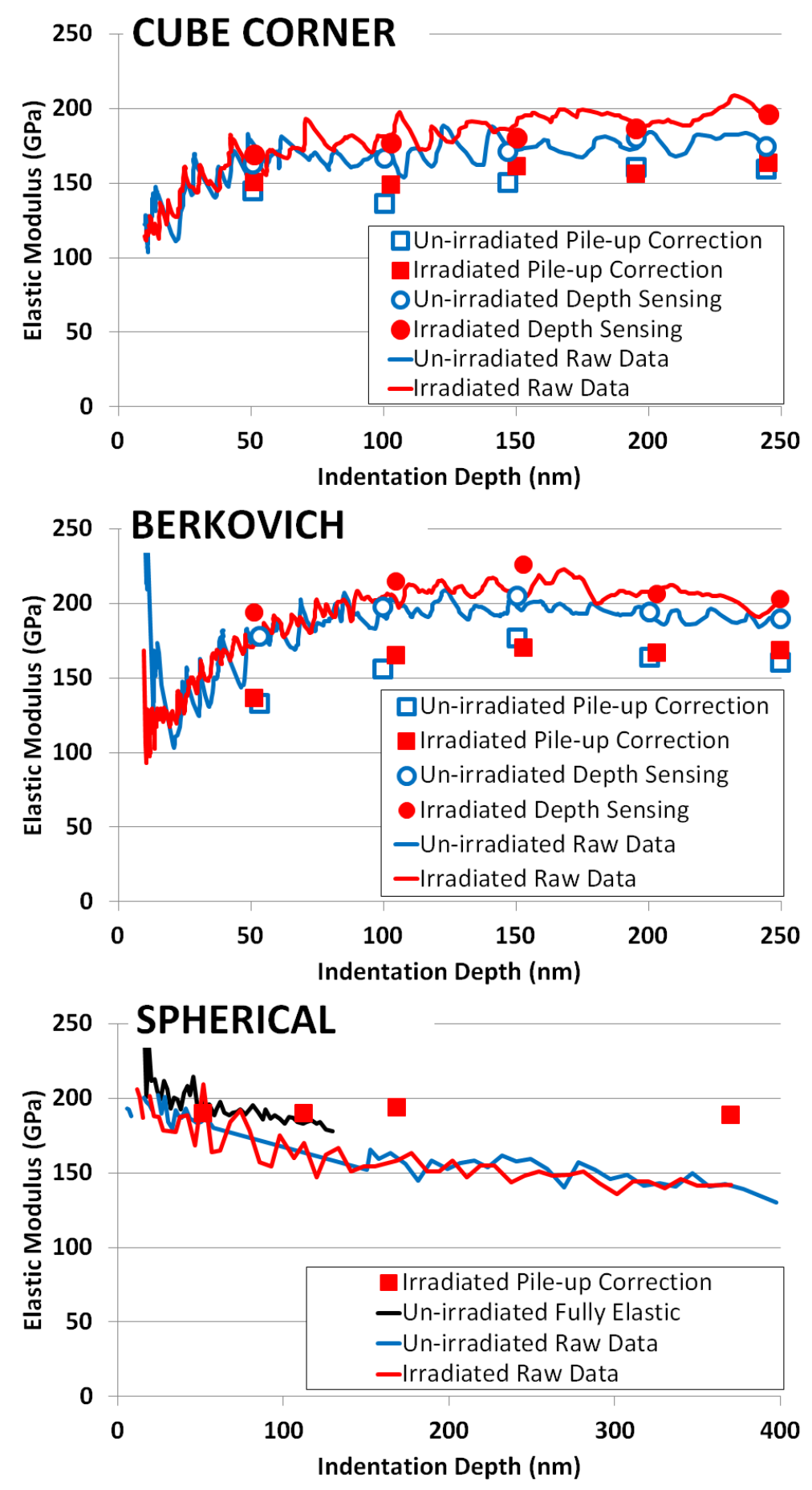

Figure 14 - Elastic modulus data produced with indentation by the cube corner, Berkovich and spherical tips into un-irradiated (blue) and irradiated (red) material. Original data plotted against data corrected for pile-up (pyramidal tips) and sink-in (spherical tip). 

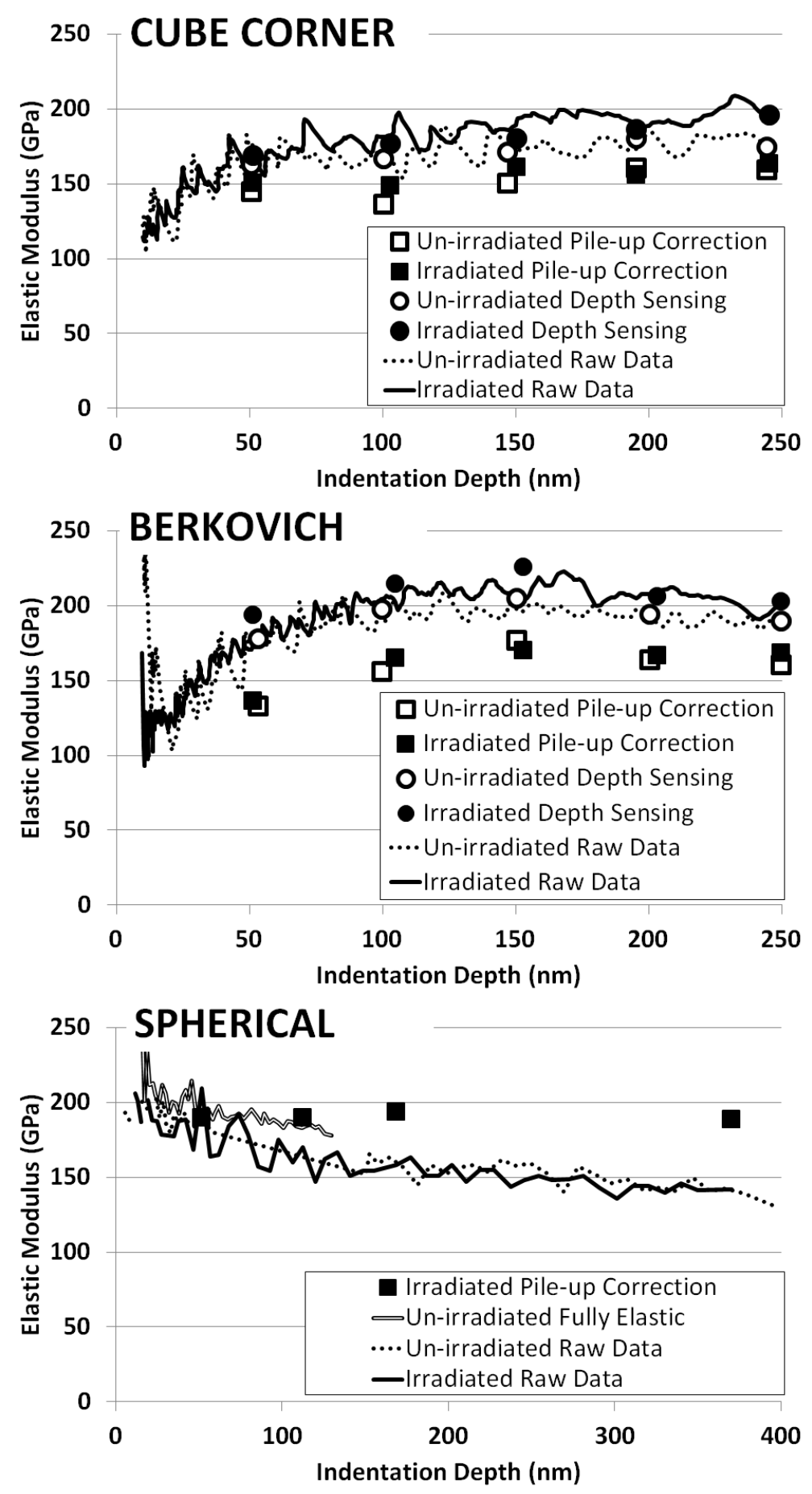

Figure 14 - Elastic modulus data produced with indentation by the cube corner, Berkovich and spherical tips into un-irradiated (dashed line/open symbols) and irradiated (solid line/sybols) material. Original data plotted against data corrected for pile-up (pyramidal tips) and sink-in (spherical tip). 

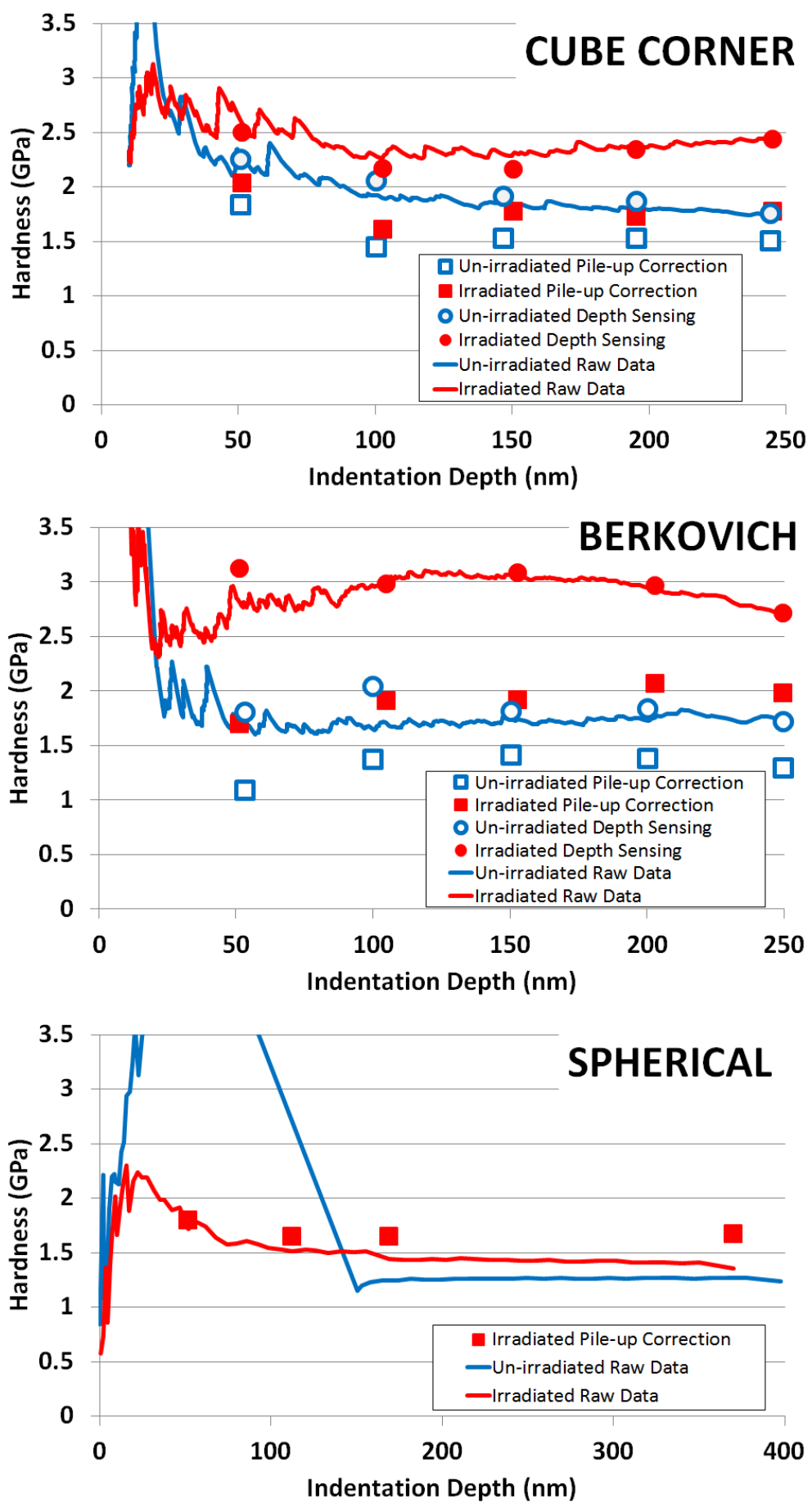

Figure 15 - Hardness data produced by indentation with the cube corner, Berkovich and spherical tips into un-irradiated (blue) and irradiated (red) material. Original data plotted against data corrected for pile-up (pyramidal tips) and sink-in (spherical tip). 

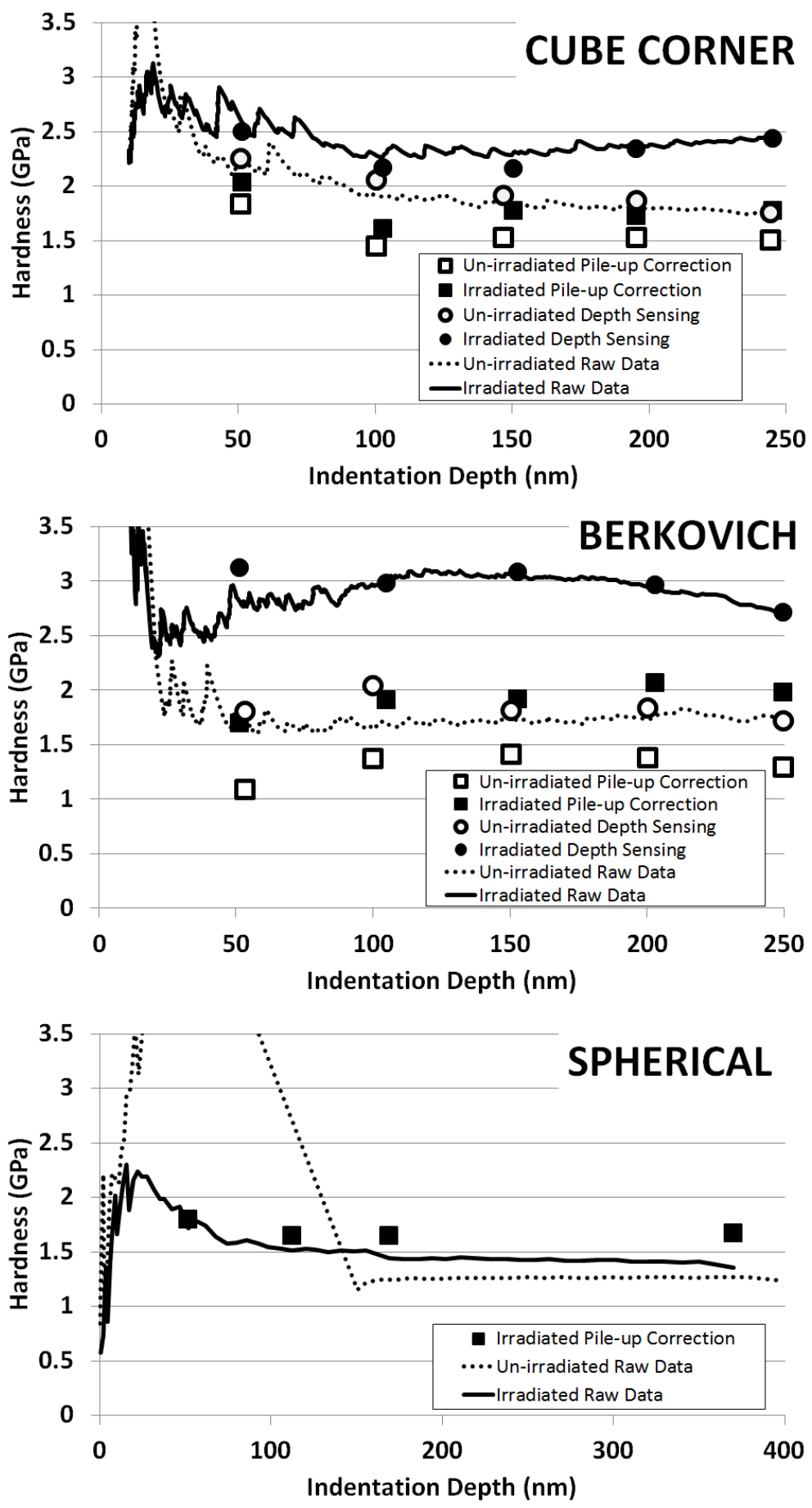

Figure 15 - Hardness data produced by indentation with the cube corner, Berkovich and spherical tips into un-irradiated (dashed line/open symbols) and irradiated (solid line/symbols) material. Original data plotted against data corrected for pile-up (pyramidal tips) and sink-in (spherical tip). 


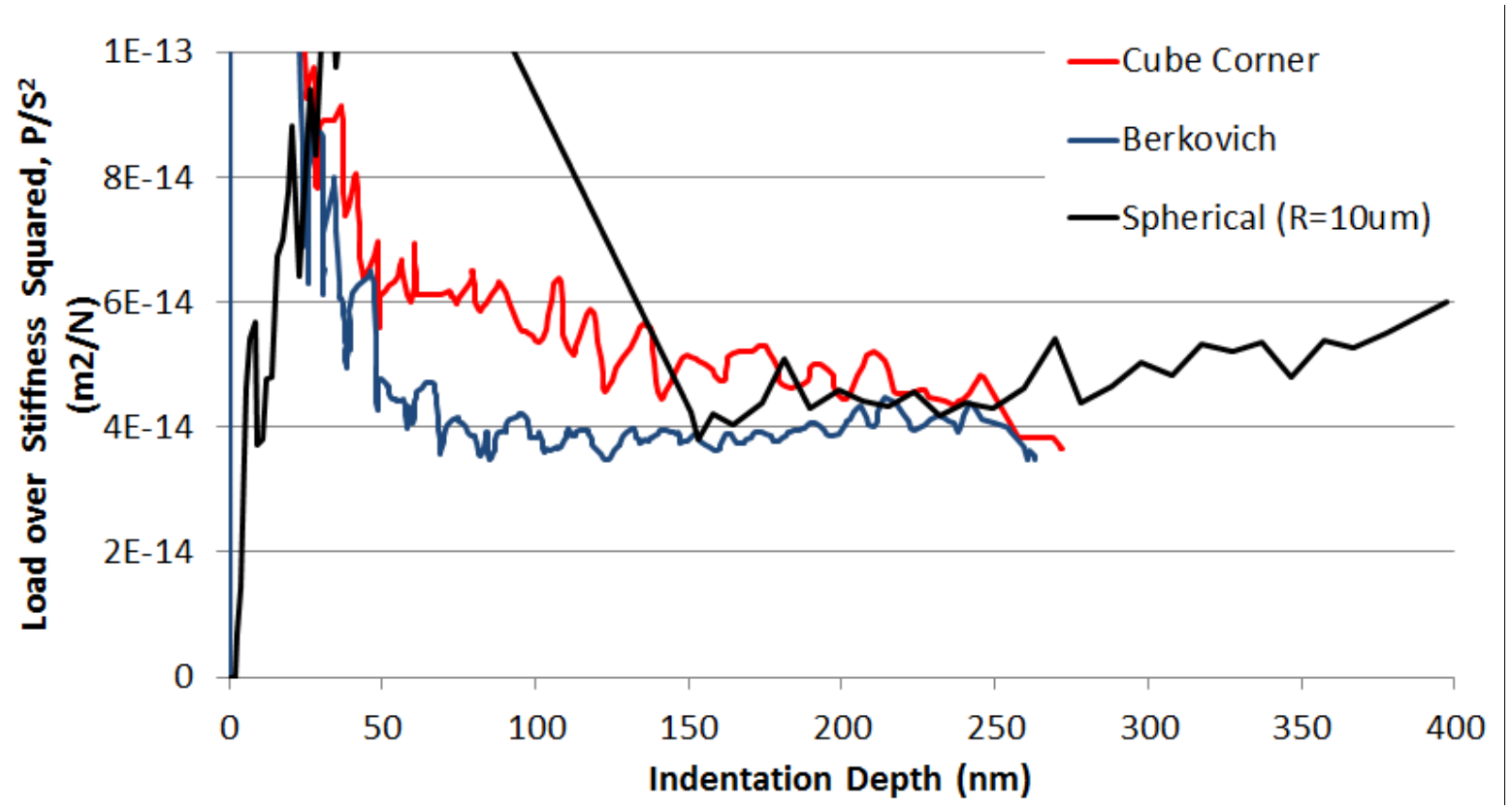

Figure 16 - Plots of load over stiffness squared versus indentation depth for indentations in the unirradiated region of the same grain, produced by the cube corner (red), Berkovich (blue) and spherical (black) tips.

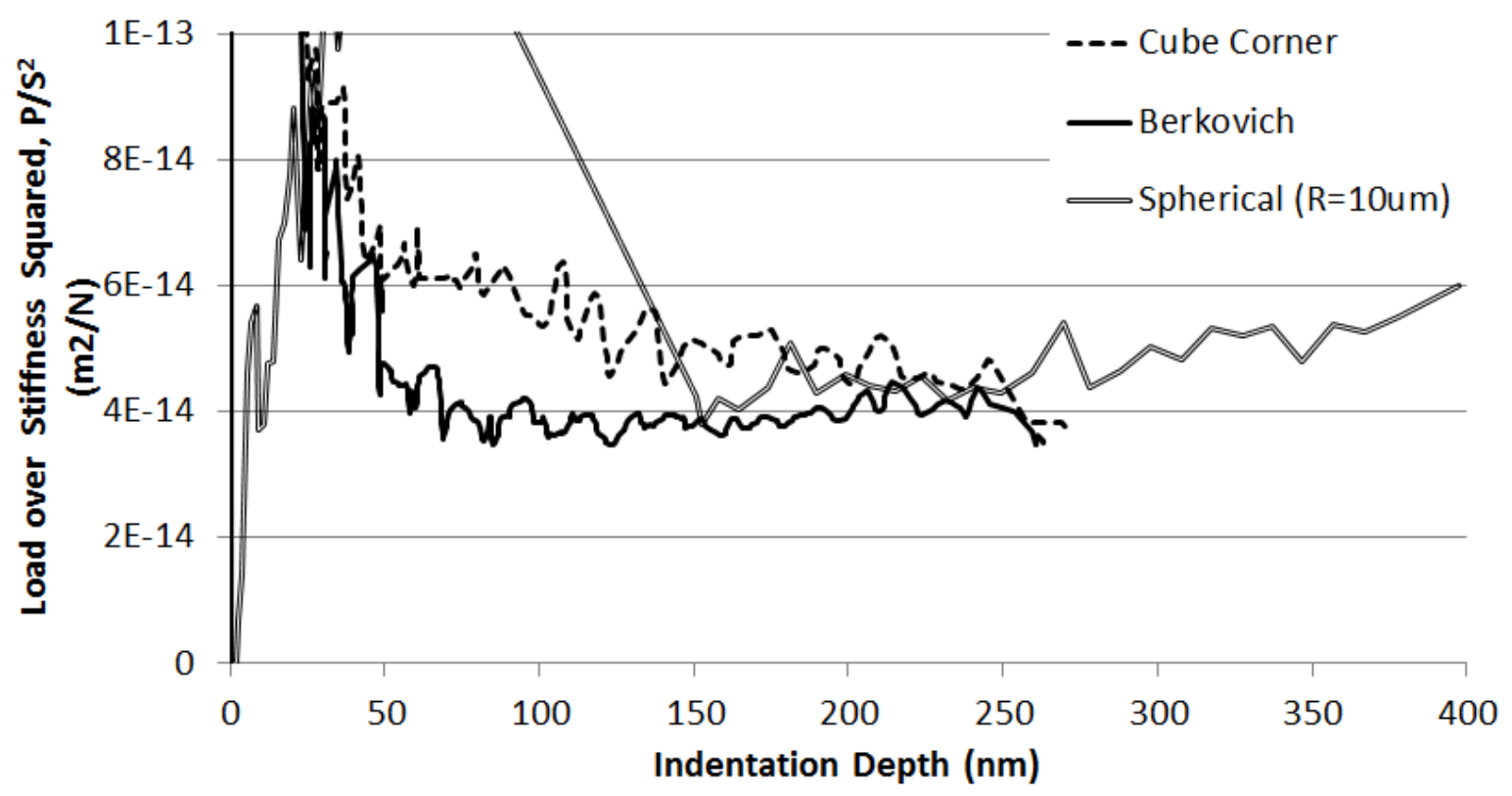

Figure 16 - Plots of load over stiffness squared versus indentation depth for indentations in the unirradiated region of the same grain, produced by the cube corner (dashed line), Berkovich (solid line) and spherical (open line) tips. 


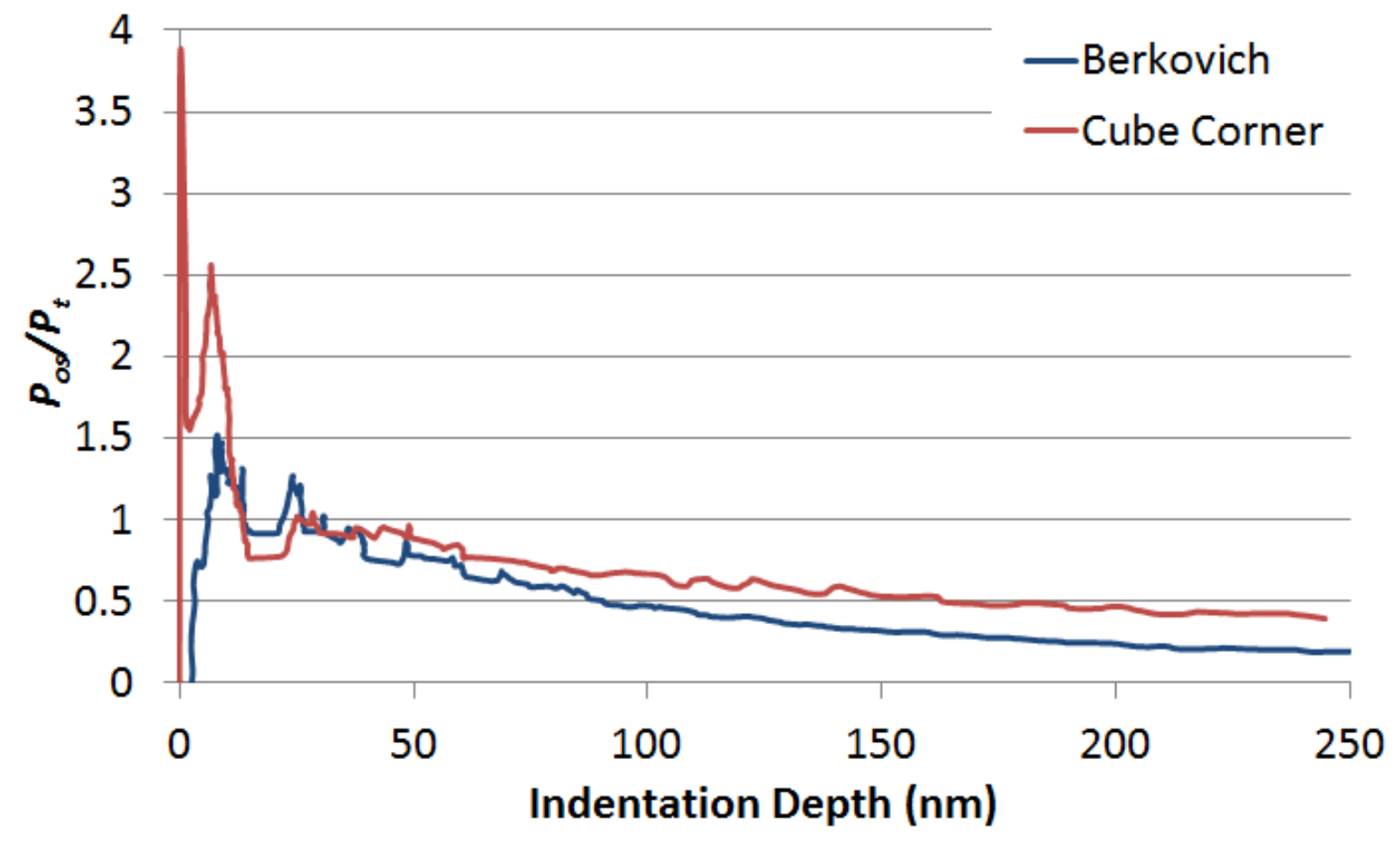

Figure 17 - Ratio of load oscillation to total load $\left(P_{o s} / P_{t}\right)$ for cube corner (red) and Berkovich (blue) tips.

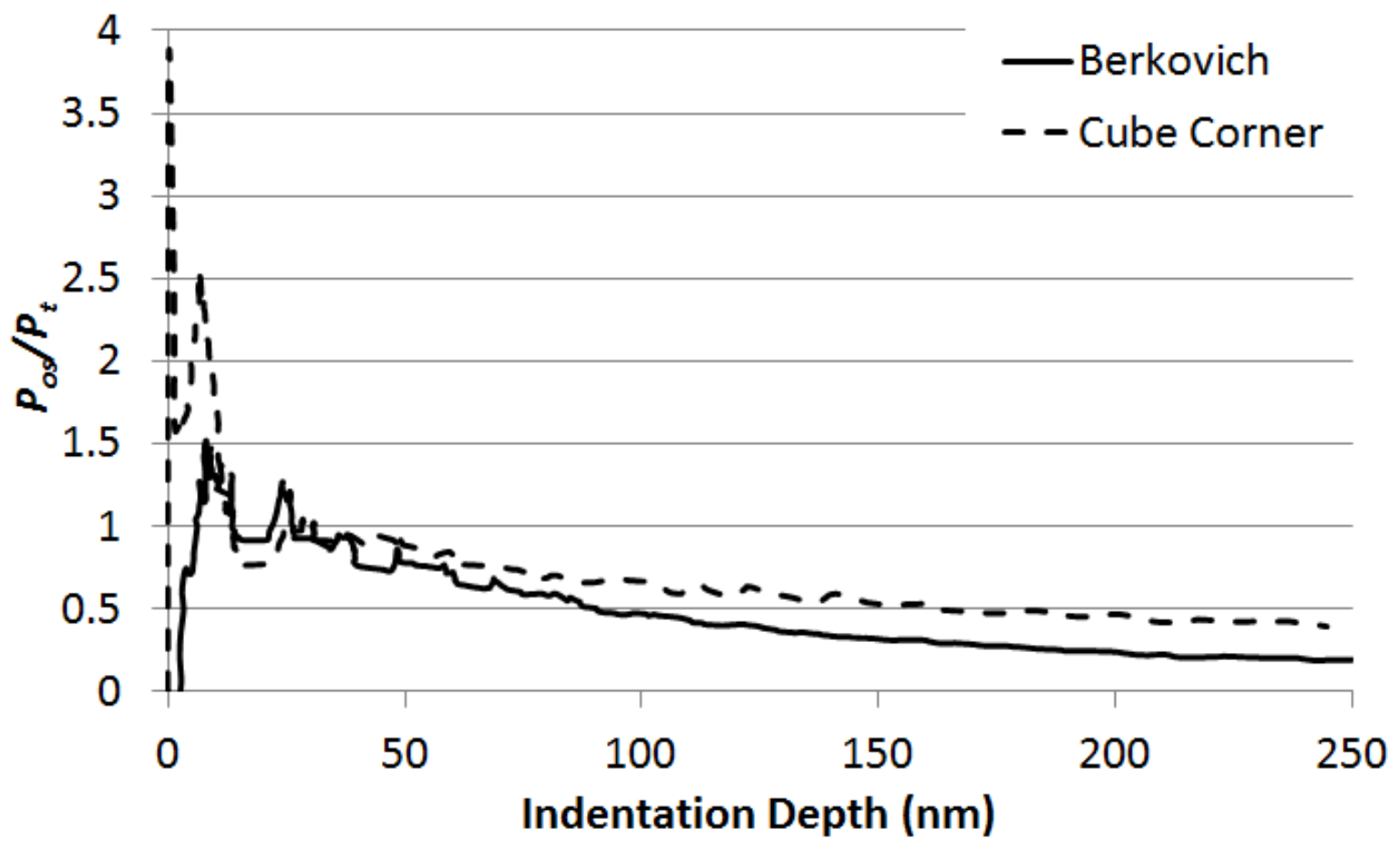

Figure 17 - Ratio of load oscillation to total load $\left(\boldsymbol{P}_{o s} / \boldsymbol{P}_{t}\right)$ for cube corner (dashed line) and Berkovich (solid line) tips. 